\title{
A Simulation Analysis and Screening of Deleterious Non-Synonymous Single Nucleotide Polymorphisms (SNPs) in Human CDKN1A Gene
}

\author{
G. M. Shazzad Hossain Prince ${ }^{1, *}$, Trayee Dhar ${ }^{1}$ \\ ${ }^{1}$ Department of Microbiology, Noakhali Science and Technology University, Noakhali, 3814, Bangladesh. \\ *Corresponding Author: shazzad.prince27@gmail.com
}

\begin{abstract}
CDKN1A also known as $p 21^{C I P I} / p 21^{W A F 1}$, a cyclin dependent kinase 1, interacts with proliferating cell nuclear antigen (PCNA) resulting in cell cycle inhibition in human. Non-synonymous single nucleotide polymorphisms (nsSNPs), which reside in the coding region of a gene, might distort the normal function of the corresponding protein. In silico analysis in this study followed many different algorithms. Following the final screening of $118 \mathrm{nsSNPs}$ from dbSNP $(\mathrm{NCBI}), 12$ missense SNPs $(\mathrm{R} 19 \mathrm{C}(\mathrm{C} \rightarrow \mathrm{T}), \mathrm{G} 23 \mathrm{D}(\mathrm{A} \rightarrow \mathrm{G}), \mathrm{V} 25 \mathrm{G}(\mathrm{G} \rightarrow \mathrm{T}), \mathrm{V} 25 \mathrm{~L}(\mathrm{C} \rightarrow \mathrm{G}), \mathrm{Q} 29 \mathrm{P}(\mathrm{A} \rightarrow \mathrm{C} \rightarrow \mathrm{G}), \mathrm{F} 51 \mathrm{~L}$ $(\mathrm{C} \rightarrow \mathrm{T})$, E56K $(\mathrm{A} \rightarrow \mathrm{G})$, T57I $(\mathrm{C} \rightarrow \mathrm{T}), \mathrm{G} 61 \mathrm{R}(\mathrm{C} \rightarrow \mathrm{G}), \mathrm{G} 61 \mathrm{D}(\mathrm{A} \rightarrow \mathrm{G}), \mathrm{Y} 151 \mathrm{C}(\mathrm{A} \rightarrow \mathrm{G})$ and $\mathrm{R} 156 \mathrm{~W}(\mathrm{C} \rightarrow \mathrm{G} \rightarrow \mathrm{T})$ were predicted to have deleterious effect by all the algorithms. Of them, R19C, G23D, F51L, Y151C and R156W occurred at the highly conserved site. G23D, F51L variants also occurred at the CDI domain. Homology structures of the protein predicted decrease of energy in mutant models. GV-GD scores predicted only two variants as neutral (V25L, F51L).
\end{abstract}

Keywords CDKN1A, p21 ${ }^{\text {cip1 }}$, nsSNPs, In silico, Cyclin dependent kinase 1

\section{Introduction}

CDKN1A or $p 21^{c i p l} / p 21^{W A F l}$ negatively regulates cell cycle at G1 check point by binding to proliferating cell nuclear antigen (PCNA) which allows cells to repair damaged DNA. Cyclin dependent kinase inhibitor 1 or $p 21^{\text {cipl }}$ inactivates PCNA. This promotes functions of $p 53$ (Yates et al., 2015). Dysfunction at G1 checkpoint may give rise to mutation and inappropriate cell proliferation which in turn might induce cancer progression (Bahl et al., 2000; Facher et al., 1997). CDKN1A or $p 21^{c i p l}$ also displays anti-apoptotic roles while inhibiting stress activated protein kinase (SAPK), apoptosis signal-regulating kinase 1 (ASK1), and inhibiting Fas-mediated apoptosis. (Yates et al., 2015). CDKN1A inhibits functional subunits of cyclinA-cdk2, cyclin E-cdk2, cyclin D-cdk4, and cyclin D-cdk6 and is unregulated by TP53 pathway (Ravitz and Wenner, 1997). It plays central role in cellular growth arrest, terminal differentiation, and apoptosis. Direct regulation of $\mathrm{p} 21$ expression occurs by $p 53$, and if $p 21^{\text {cipl } 1} / p 21^{\text {WAFl }}$ is cleaved by Caspase-3-mediated pathway, apoptosis of cancer cell follows (Ralhan et al., 2000). Mutations in TP53 is found in association with chronic lymphocytic leukemia (CLL). Dysfunction in the interaction between TP53 and CDKN1A can cause structural abnormality of TP53 (Tracy et al., 2017).

Some common sequence variants, such as polymorphism on codon 31 in $\mathrm{p} 21$, was found associated with the procession of breast cancer. Mutation in CDKN1A, commonly known as or $\mathrm{p} 21^{\mathrm{cip} 1} / \mathrm{p} 21^{\mathrm{WAF} 1}$, was hardly responsible for various types of cancers, despite being a principle downstream regulator of TP53 (Powell et al., 2002). But two common polymorphisms, $\mathrm{C} \rightarrow \mathrm{A}$ transversion at codon $31(\mathrm{Ser} \rightarrow \mathrm{Arg}$ ) and $\mathrm{C} \rightarrow \mathrm{T}$ transition in the 3'UTR of exon 3, 20bp downstream from strop codon) in CDKN1A or $\mathrm{p} 21^{\mathrm{cip} 1} / \mathrm{p} 21^{\mathrm{WAF} 1}$ has been suggested to induce development of some types of cancer i.e. esophageal cancer. (Bahl et al., 2000; Facher et al., 1997; Powell et al., 2002; Tracy et al., 2017). 
Another polymorphism $\mathrm{A} \rightarrow \mathrm{G}$ at codon 149 (Asp $\rightarrow \mathrm{Gly}$ ) was observed in esophageal squamous cell carcinomas (ESCCs) in Indian patients and was predicted to interfere with $p 53$ pathway as well as esophageal tumorigenesis (Bahl et al., 2000; Powell et al., 2002). Polymorphism in CDKN1A was also reported as a risk factor to Alzheimer's disease (AD) (Yates et al., 2015). In silico methods with great accuracy was also followed to screen disease associated nsSNPs in many studies using computational tool such as- PolyPhen 2.0, SIFT, PANTHER, I-mutant 3.0, PhD-SNP, SNP\&GO, Pmut, and Mutpred etc. (Ali et al., 2017; Kamaraj and Purohit, 2013; Mathe et al., 2006; Stojiljkovic et al., 2016). Transactivation activity of 1514 missense substitution was analyzed by a Align GV-GD algorithm which scores substitution from C0 (neutral) to C65 (deleterious) (Ali et al., 2017; Mathe et al., 2006). Conservation analysis, based on the evolutionary information, of a protein unfolds its amino acid positions along the chain, which is essential to retain its structural integrity and function (Ashkenazy et al., 2016). Many different computer based algorithms to predict missense variants should be optimized as well as sequence alignment to find the best result (Hicks et al., 2011). Application of many computational analysis was employed in this study to find deleterious nsSNPs of CDKN1A as well as their functional effect on the corresponding protein.

\section{Methods}

\subsection{Collecting SNPs and Protein's Sequence from the databases}

Human CDKN1A gene SNPs were collected from the National Center for Biological Information (NCBI) online dbSNP database (https://www.ncbi.nlm.nih.gov/snp). From this server, the non-synonymous SNP (nsSNP) information (Chromosome coordinates and alleles) was retrieved for the computational analysis in this study (Sherry et al., 2001). Protein IDs from the analysis of nsSNPs in SIFT server (http://sift.jcvi.org/) were used to retrieve FASTA sequence of the protein encoded by Human CDKN1A gene from the Ensemble database (https://ensembl.org/). Ensemble is a growing database which has been working for aggregating, processing, integrating and redistributing genomic datasets (Zerbino et al., 2017).

\subsection{Deleterious SNPs predicted by SIFT and Polyphen-2}

Sorting intolerant from the tolerant (SIFT) server was used to predict the damaging nsSNPs (Kumar et al., 2009). It receives a query sequence and employs multiple alignment to predict either tolerated or damaging substitution in each position of the query sequence ( $\mathrm{Ng}$ and Henikoff, 2003). nsSNPs with a normalized probability score less than 0.05 is predicted to be harmful and a score greater than 0.05 is considered as tolerated ( $\mathrm{Ng}$ and Henikoff, 2006). Polyphen2 server available at (http://genetics.bwh.harvard.edu/pph2/) was utilized, using the data gathered from SIFT result of the nsSNPs, to find either deleterious or neutral amino acid substitutions. By naive Bayesian classifier, it identifies the potential amino acid substitution and the effect of mutation (Adzhubei et al., 2010). It classifies SNPs as "Probably damaging" (0.85 to 1.00), "Possibly damaging' (0.15 to 0.84) and "Benign" (<0.14) (Ali et al., 2017).

\subsection{Disease related amino acid substitution by Predict-SNP, MAPP, PhD-SNP, and SNAP}

Predict SNP, a consensus classifiers for prediction of disease related amino acid mutation, available at (https://loschmidt.chemi.muni.cz/predictsnp1/) with integrated tools- MAPP, PhD-SNP 2.06 and SNAP 1.1.30, was used to determine the deleterious or neutral nsSNPs (Bendl et al., 2014). Multivariate Analysis of Protein Polymorphism (MAPP) defines deleterious nsSNPs based on the physicochemical variation present in a column of protein sequence akignment (Stone and Sidow, 2005). Predictor of Human Deleterious Single Nucleotide Polymorphism (PhD-SNP) is a Support Vector Machine (SVM) which uses evolutionary information to sort out SNPs related to Mendelian and complex diseases from the neutral ones (Capriotti et al., 2006). SNAP screens out nonacceptable polymorphisms using neural network. It determines $80 \%$ of the deleterious substitution at $77 \%$ accuracy and $76 \%$ of the neutral substitutions at $80 \%$ accuracy (Bromberg and Rost, 2007).

\subsection{Analysis of protein's stability change upon amino acid substitution}




\subsubsection{I-Mutant 3.0 server}

A Support Vector Machine (SVM) based predictor I-Mutant 3.0, available at (http://gpcr2.biocomp.unibo.it/cgi/predictors/I-Mutant3.0/I-Mutant3.0.cgi, was used in this study to calculate the change in the stability of protein resulting from amino acid substitution (Capriotti et al., 2005). It computes the DDG $(\mathrm{kcal} / \mathrm{mol})$ value and RI value (reliability index) of a submitted mutation of a protein.

\subsubsection{MUpro server}

MUpro server uses both the Support Vector Machine (SVM) and neural network to predict the change in the stability of the protein upon single site mutation. It predicts, with an $84 \%$ accuracy, amino acid substitutions responsible for either decreasing or increasing the stability of the related protein via cross validation methods on a large dataset of single amino acid mutations. No tertiary structure of the protein is required for the prediction. (Cheng et al., 2006). MUpro web server available at (http://mupro.proteomics.ics.uci.edu/) was used in this study and a confidence score $<0$ was predicted to cause decrease in the stability and a score $>0$ is predicted to cause increase in the stability.

\subsection{Structural conformation and conservation analysis by ConSurf sever}

Highly conserved functional regions of the protein coded by CDKN1A gene was identified by ConSurf tool (http://consurf.tau.ac.il/). It constructs a phylogenetic tree, following multiple sequence alignment of a query sequence, to run a high-throughput identification process of the functional regions of a protein based on evolutionary data (Ashkenazy et al., 2016).

\subsection{Prediction of secondary structure by PSIPRED}

Secondary structure of CDKN1A was predicted using PSIPRED server available at (http://bioinf.cs.ucl.ac.uk/psipred/) (Buchan et al., 2013). It is based on a two stage neural network with the implementation of position specific scoring matrices constructed from PSI-BLAST to predict the available secondary structures of a protein. (Jones, 1999).

\subsection{Homology Modelling}

3D structures of the protein encoded by CDKN1A was constructed using four different homology modelling tools as no crystal structure with appropriate length of this protein was available in protein data bank.

\subsubsection{Homology modelling by Muster server}

MUlti-score ThreadER (MUSTER) algorithm (https://zhanglab.ccmb.med.umich.edu/MUSTER/), with the implementation of MODELLER v8.2 to construct full length protein model following sequence-template alignment, was used in this study. It uses information such as- sequence profiles, secondary structures, structure fragment profiles, solvent accessibility, dihedral torsion angles, hydrophobic scoring matrix of different sequences to construct the model (Wu and Zhang, 2008). It provides several template based models with a scoring function expressed as $\mathrm{Z}$ value to determine the best model.

\subsubsection{Homology modelling by Phyre-2 server}

Phyre-2 tool (http://www.sbg.bio.ic.ac.uk/phyre2/), based on Hidden Markov method, was used to predict the homology based three dimensional structure of the query amino acid sequence. It combines four steps to build a model: (1) collecting homologous sequence, (2) screening of fold library, (3) loop modelling, (4) multiple template modelling by ab-initio folding simulation-Poing and (4) Side chain placement (Kelley et al., 2015).

\subsubsection{Homology modelling by RaptorX server}

RaptorX server available at (http://raptorX.uchicago.edu/StructurePrediction/predict/), using RaptorX-Boost and RaptorX-MSA to construct three dimensional structure of a protein, predicted the 3D model of the protein coded by 
CDKN1A. It combines a nonlinear scoring function and a probabilistic-consistency algorithm for predicting the model structure (Källberg et al., 2012).

\subsubsection{Homology modelling by Swiss-Model server}

Swiss-Model workplace available at (https://swissmodel.expasy.org/), a web based tool for the homology modelling of protein, was used to avail the three dimensional structure of CDKN1A (Arnold et al., 2006). The accuracy of the constructed model is calculated by CAMEO system. Swiss-model is an automated tool based on evolutionary information which searches for the best sequence-template alignment from its high-throughput template library (SMTL) to build the model (Biasini et al., 2014).

\subsection{Model Refinement, energy minimization and mutation}

High resolution structure refinement of the protein model at the atomic level was carried out by ModRefiner tool (Xu and Zhang, 2011) and energy minimization executed for the improvement of the quality of models with the GROMOS 96 forcefield (van Gunsteren, 1996) implementation of DeepView v.4.1.0 (swiss pdb viewer) (Guex and Peitsch, 1997). Execution of energy minimization was done in vacao without any reaction field. Swiss PdbViewer v.4.1.0 was also implemented to build mutated models by browsing a rotamer library of the protein model. Energy minimization of mutant models was brought about to increase the quality.

\subsection{Visualization of different models by BIOVIA Discovery Studio Visualizer 2016}

Discovery Studio Visualizer (Dassault Systèmes, 2016) tool was used to display the 3D structures of the constructed models following structure refinement and energy minimization.

\subsection{Validation of models}

\subsubsection{Ramachandran plot analysis}

Ramachandran plot analysis of the protein models, available at ((http://www-cryst.bioc.cam.ac.uk/rampage) determined the energetically allowed sites of amino acid residues in a protein structure through the calculation of backbone dihedral angles of $\psi$ against $\varphi$ of amino acid residues (Lovell et al., 2003).

\subsubsection{QMEAN6 Z-value}

The degree of nativeness of the predicted models are evaluated by the QMEAN scoring (https://swissmodel.expasy.org/qmean/). The major five geometrical features of the protein is predicted by this composite scoring function (Benkert et al., 2008).

\subsection{Screening of most frequent deleterious substitution}

IMB SPSS Statistics for Windows v.20 (IBM Corp., 2011) was used to find the most frequent variants having deleterious effect based on the prediction of six evaluation methods- Predict SNP, MAPP, PhD SNP, SNAP, I-Mutant 3.0 and Mupro, following the initial screening by SIFT and Polyphen-2, which predicted 53 nsSNPs as being harmful out of 118 .

\subsection{Visualization of selected mutations using Mutation 3D server}

Mutation 3D server (http://mutation3d.org/), an interactive tool to visualize amino acid substitutions along with their position on functional domains, was employed in this study. The algorithm used here is an approach based on 3D clustering method and was used to predict driver genes in cancer (Meyer et al., 2016).

\subsection{Prediction of biophysical activity by Align-GVGD}

Align-GVGD predicted the biophysical characteristics of the amino acid substitutions (http://agvgd.hci.utah.edu/). Grantham Variation (GV) and Grantham Deviation (GD) scores (0 to >200) and graded classifiers (C0 to C65) were 
calculated to predict either deleterious or neutral variants (Mathe et al., 2006). An extension of Grantham difference to multiple sequence alignments and true simultaneous multiple comparisons, Align-GVGD, predicts missense substitutions (Tavtigian et al., 2006).

\subsection{Harmful effects of selected variants by MutPred2 server}

MutPred2 (http://mutpred2.mutdb.org/) web server proposed the reasons behind the disease related amino acid substitution at the molecular level (Pejaver et al., 2017). The result generated by this algorithm predicts a general probability score to define a substitution as deleterious or disease related and includes top five molecular features with a P-value. Scores are interpreted in three categories- actionable hypotheses (general probability score, $g>0.5$ and property score, $p<0.05)$, confident hypotheses $(g>0.75$ and $p<0.05)$, and very confident hypotheses $(g>0.75$ and $p<0.01$ ) (Li et al., 2009).

\section{Results}

NCBI dbSNP database contains information on SNPs of various genes. Mining this database on December 11, 2017, revealed 1766 SNPs of CDKN1A gene, of which 1718 were from Homo sapiens. Of the 118 CDKN1A nonsynonymous SNPs found in humans, 113 were missense mutation and the rest 5 were nonsense mutation. FASTA format of the protein encoded by CDKN1A gene, retrieved from Ensemble database, is 164 amino acid long.

\subsection{Prediction of functional context of nsSNPs}

Functional features of the 118 CDKN1A nsSNPs analyzed by SIFT and Polyphen-2 screened out 65 nsSNPs as because they were predicted as "Tolerated" by SIFT and "Benign" by Polyphen-2 server. All 5 nonsense mutations were found to be either tolerated or benign by these servers.

Table 1 represents the final scores of 53 nsSNPs generated by the sequence homology based software SIFT and Polyphen-2. In SIFT, $23 \mathrm{nsSNPs}$ out of $53 \mathrm{had}$ a tolerance index $\leq 0.05$ and predicted as "Damaging". While analyzing with Polyphen-2, two score were calculated-HumDiv and HumVar. Polyphen-2 gives score ranging from 0 (neutral) to 1 (damaging). HumDiv predicted 34 nsSNPs as "Probably Damaging (High Confidence)" and 12 as "Possibly Damaging (Low confidence)" out of 53 nsSNPs. HumVar reported 28 nsSNPs as "Probably Damaging (High Confidence)" and 10 as "Possibly Damaging (Low confidence)" out of 53 nsSNPs.

\begin{tabular}{lllllllllll}
\hline \multirow{2}{*}{ SNP ID } & \multirow{2}{*}{ Protein Id } & Allele & Variant & \multicolumn{2}{c}{ Polyphen-2 } & & \multicolumn{2}{c}{ SIFT } \\
\cline { 5 - 9 } & & & & HumDiv & Value & HumVar & Value & SIFT Prediction & Score \\
\hline rs148679597 & ENSP00000244741 & A/G & C117Y & Pro. D & 0.995 & Pro. D & 0.976 & Damaging & 0.05 \\
\hline rs4986867 & ENSP00000244741 & A/C/T & F63L & Pos. D & 0.749 & Pos. D & 0.492 & Tolerated & 0.31 \\
\hline rs12721594 & ENST00000244741 & A/G & R48Q & Pro. D & 1 & Pro. D & 0.961 & Damaging & 0.03 \\
\hline rs45548832 & ENSP00000244741 & A/G/T & R67H & Pro. D & 0.999 & Pro. D & 0.927 & Tolerated & 0.09 \\
\hline rs114149607 & ENSP00000244741 & C/T & T80M & B & 0.112 & B & 0.002 & Damaging & 0.3 \\
\hline rs151314631 & ENSP00000244741 & C/G & V25L & Pro. D & 0.999 & Pro. D & 0.997 & Tolerated & 0.22 \\
\hline rs199801759 & ENSP00000244741 & A/G & Y151C & Pro. D & 1 & Pro. D & 0.997 & Damaging & Tolerated \\
\hline
\end{tabular}


bioRxiv preprint doi: https://doi.org/10.1101/240820; this version posted January 3, 2018. The copyright holder for this preprint (which was not certified by peer review) is the author/funder, who has granted bioRxiv a license to display the preprint in perpetuity. It is made available under aCC-BY-NC-ND 4.0 International license.

\begin{tabular}{|c|c|c|c|c|c|c|c|c|c|}
\hline rs368128681 & ENSP00000244741 & $\mathrm{A} / \mathrm{G}$ & G23D & Pro. D & 0.998 & Pro. D & 0.983 & Damaging & 0 \\
\hline rs369108178 & ENSP00000244741 & $\mathrm{C} / \mathrm{T}$ & P134L & $\mathrm{B}$ & 0.046 & B & 0.03 & Damaging & 0.04 \\
\hline rs370291979 & ENSP00000244741 & $\mathrm{A} / \mathrm{G}$ & T57A & Pro. D & 0.995 & Pro. D & 0.953 & Tolerated & 0.56 \\
\hline rs371219528 & ENSP00000244741 & $\mathrm{C} / \mathrm{T}$ & $\mathrm{S} 2 \mathrm{P}$ & Pos. D & 0.838 & $\mathrm{~B}$ & 0.446 & Tolerated & 0.16 \\
\hline rs372390764 & ENSP00000244741 & $\mathrm{C} / \mathrm{T}$ & $\mathrm{R} 67 \mathrm{C}$ & Pro. D & 0.999 & Pro. D & 0.927 & Damaging & 0.04 \\
\hline rs372882329 & ENSP00000244741 & $\mathrm{C} / \mathrm{T}$ & L113P & Pro. D & 0.999 & Pro. D & 0.987 & Tolerated & 0.29 \\
\hline rs 373450720 & ENSP00000244741 & $\mathrm{C} / \mathrm{T}$ & $\mathrm{R} 32 \mathrm{C}$ & Pro. D & 0.999 & Pro. D & 0.928 & Damaging & 0.01 \\
\hline rs374154006 & ENSP00000244741 & $\mathrm{C} / \mathrm{G} / \mathrm{T}$ & R156W & Pro. D & 1 & Pro. D & 0.998 & Damaging & 0 \\
\hline rs374965936 & ENSP00000244741 & $\mathrm{C} / \mathrm{T}$ & R83W & B & 0.001 & B & 0.001 & Damaging & 0.02 \\
\hline rs375050346 & ENSP00000244741 & $\mathrm{A} / \mathrm{C} / \mathrm{G}$ & $\mathrm{R} 20 \mathrm{H}$ & Pos. D & 0.894 & Pos. D & 0.621 & Tolerated & 0.22 \\
\hline \multirow[t]{2}{*}{ rs376481017 } & ENSP00000244741 & $\mathrm{A} / \mathrm{C} / \mathrm{G}$ & D33N & Pro. D & 1 & Pro. D & 0.994 & Tolerated & 0.38 \\
\hline & ENSP00000244741 & $\mathrm{A} / \mathrm{C} / \mathrm{G}$ & D33H & Pro. D & 1 & Pro. D & 0.996 & Tolerated & 0.2 \\
\hline rs541505866 & ENSP00000244741 & $\mathrm{C} / \mathrm{G}$ & D52E & Pro. D & 0.998 & Pro. D & 0.976 & Damaging & 0.01 \\
\hline rs559151286 & ENSP00000244741 & $\mathrm{C} / \mathrm{T}$ & A39V & Pos. D & 0.947 & Pos. D & 0.518 & Tolerated & 0.25 \\
\hline rs569995916 & ENSP00000244741 & $\mathrm{A} / \mathrm{G}$ & G104R & Pos. D & 0.952 & Pos. D & 0.621 & Tolerated & 0.48 \\
\hline \multirow[t]{2}{*}{ rs746709171 } & ENSP00000244741 & $\mathrm{A} / \mathrm{C} / \mathrm{T}$ & $\mathrm{A} 36 \mathrm{E}$ & Pos. D & 0.810 & Pos. D & 0.49 & Tolerated & 1 \\
\hline & ENSP00000244741 & $\mathrm{A} / \mathrm{C} / \mathrm{T}$ & $\mathrm{A} 36 \mathrm{~V}$ & Pro. D & 0.974 & Pos. D & 0.673 & Tolerated & 0.26 \\
\hline rs748758330 & ENSP00000244741 & $\mathrm{C} / \mathrm{T}$ & R69W & $\mathrm{B}$ & 0.004 & B & 0.005 & Damaging & 0.04 \\
\hline rs748842488 & ENSP00000244741 & $\mathrm{C} / \mathrm{T}$ & F51L & Pro. D & 0.999 & Pro. D & 0.998 & Damaging & 0.01 \\
\hline rs750249677 & ENSP00000244741 & $\mathrm{C} / \mathrm{T}$ & $\mathrm{R} 155 \mathrm{C}$ & Pro. D & 1 & Pro. D & 0.981 & Damaging & 0 \\
\hline rs752126483 & ENSP00000244741 & $\mathrm{A} / \mathrm{C}$ & D112E & Pos. D & 0.884 & B & 0.433 & Tolerated & 1 \\
\hline rs752557277 & ENSP00000244741 & $\mathrm{C} / \mathrm{T}$ & $\mathrm{R} 9 \mathrm{C}$ & $\mathrm{B}$ & 0.004 & B & 0.002 & Damaging & 0.02 \\
\hline rs752792828 & ENSP00000244741 & $\mathrm{C} / \mathrm{T}$ & $\mathrm{Y} 77 \mathrm{H}$ & Pos. D & 0.61 & $\mathrm{~B}$ & 0.28 & Damaging & 0.03 \\
\hline rs753291170 & ENSP00000244741 & $\mathrm{A} / \mathrm{G}$ & E56K & Pro. D & 0.999 & Pro. D & 0.926 & Tolerated & 0.18 \\
\hline rs753529000 & ENSP00000244741 & $\mathrm{C} / \mathrm{T}$ & $\mathrm{R} 122 \mathrm{C}$ & Pro. D & 0.978 & $\mathrm{~B}$ & 0.353 & Tolerated & 0.24 \\
\hline \multirow[t]{2}{*}{ rs756383281 } & ENSP00000244741 & $\mathrm{A} / \mathrm{C} / \mathrm{G}$ & Q29P & Pro. D & 0.958 & Pos. D & 0.802 & Damaging & 0.01 \\
\hline & ENSP00000244741 & $\mathrm{A} / \mathrm{C} / \mathrm{G}$ & Q29R & Pos. D & 0.762 & Pos. D & 0.478 & Tolerated & 0.08 \\
\hline rs758877354 & ENSP00000244741 & $\mathrm{C} / \mathrm{G}$ & G61R & Pro. D & 0.999 & Pro. D & 0.988 & Damaging & 0 \\
\hline rs760466052 & ENSP00000244741 & $\mathrm{C} / \mathrm{T}$ & S98L & Pos. D & 0.845 & B & 0.421 & Tolerated & 0.3 \\
\hline rs762978904 & ENSP00000244741 & $\mathrm{C} / \mathrm{T}$ & $\mathrm{R} 46 \mathrm{C}$ & $\mathrm{B}$ & 0.015 & $\mathrm{~B}$ & 0.006 & Damaging & 0.02 \\
\hline
\end{tabular}


bioRxiv preprint doi: https://doi.org/10.1101/240820; this version posted January 3,2018 . The copyright holder for this preprint (which was not certified by peer review) is the author/funder, who has granted bioRxiv a license to display the preprint in perpetuity. It is made available under aCC-BY-NC-ND 4.0 International license.

\begin{tabular}{|c|c|c|c|c|c|c|c|c|c|}
\hline rs763967941 & ENSP00000244741 & $\mathrm{A} / \mathrm{G}$ & E28K & Pro. D & 1 & Pro. D & 0.999 & Tolerated & 0.1 \\
\hline rs765248879 & ENSP00000244741 & $\mathrm{G} / \mathrm{T}$ & V25G & Pro. D & 1 & Pro. D & 0.999 & Tolerated & 0.1 \\
\hline rs765503766 & ENSP00000244741 & $\mathrm{C} / \mathrm{G}$ & D33E & Pro. D & 0.998 & Pro. D & 0.968 & Tolerated & 0.77 \\
\hline rs767974221 & ENSP00000244741 & $\mathrm{G} / \mathrm{T}$ & L115R & Pro. D & 0.999 & Pro. D & 0.983 & Tolerated & 0.52 \\
\hline rs769049147 & ENSP00000244741 & $\mathrm{A} / \mathrm{G}$ & G70D & Pro. D & 0.986 & Pos. D & 0.684 & Tolerated & 0.43 \\
\hline rs769229553 & ENSP00000244741 & $\mathrm{C} / \mathrm{T}$ & R19C & Pro. D & 1 & Pro. D & 1 & Damaging & 0 \\
\hline rs770464093 & ENSP00000244741 & $\mathrm{A} / \mathrm{G}$ & $\mathrm{G} 23 \mathrm{~S}$ & Pro. D & 0.999 & Pro. D & 0.979 & Damaging & 0.01 \\
\hline rs771753909 & ENSP00000244741 & $\mathrm{C} / \mathrm{G}$ & L71V & Pro. D & 0.939 & B & 0.433 & Tolerated & 0.28 \\
\hline rs772419969 & ENSP00000244741 & $\mathrm{C} / \mathrm{T}$ & T57I & Pro. D & 1 & Pro. D & 0.99 & Tolerated & 0.22 \\
\hline rs778199042 & ENSP00000244741 & $\mathrm{A} / \mathrm{G}$ & G61D & Pro. D & 0.997 & Pro. D & 0.977 & Tolerated & 0.06 \\
\hline rs779063779 & ENSP00000244741 & $\mathrm{A} / \mathrm{G}$ & R156Q & Pro. D & 1 & Pro. D & 0.991 & Damaging & 0.02 \\
\hline rs779250316 & ENSP00000244741 & $\mathrm{A} / \mathrm{G}$ & $\mathrm{R} 19 \mathrm{H}$ & Pro. D & 1 & Pro. D & 0.999 & Damaging & 0 \\
\hline rs779717089 & ENSP00000244741 & $\mathrm{C} / \mathrm{T}$ & R86W & $\mathrm{B}$ & 0.035 & $\mathrm{~B}$ & 0.005 & Damaging & 0.01 \\
\hline rs780909704 & ENSP00000244741 & $\mathrm{A} / \mathrm{G}$ & A64T & Pos. D & 0.898 & B & 0.207 & Tolerated & 0.51 \\
\hline rs935843132 & ENSP00000244741 & $\mathrm{A} / \mathrm{C}$ & K75Q & Pos. D & 0.917 & B & 0.446 & Tolerated & 0.55 \\
\hline
\end{tabular}

Table 1. Screening of Deleterious Single Nucleotide Polymorphism (SNP) Predicted by SIFT and Polyphen-2. ${ }^{\text {a }}$ Probably damaging (more confident); ${ }^{\mathrm{b}}$ Possibly damaging (less confident); ${ }^{\mathrm{c}}$ Benign.

\subsection{Prediction of deleterious and disease related amino acid substitutions}

Predict-SNP is a consensus classifiers for the prediction of disease related mutations. Analysis of 53 of nsSNPs by this server also predicted MAPP, PhD-SNP and SNAP scores of nsSNPs. All results are summarized in

Table 2. Out of this 53 nsSNPs, 31, 50, 19 and 29 were predicted as "Deleterious" by Predict-SNP, MAPP, PhD-SNP and SNAP respectively.

\begin{tabular}{lllllllll}
\hline \multirow{2}{*}{ Variant } & $\begin{array}{l}\text { Predict- } \\
\text { SNP } \\
\text { Prediction }\end{array}$ & $\begin{array}{l}\text { Predict- } \\
\text { SNP } \\
\text { Expected } \\
\text { Accuracy }\end{array}$ & $\begin{array}{l}\text { MAPP } \\
\text { Prediction }\end{array}$ & $\begin{array}{l}\text { MAPP } \\
\text { Expected } \\
\text { Accuracy }\end{array}$ & $\begin{array}{l}\text { PhD-SNP } \\
\text { Prediction }\end{array}$ & $\begin{array}{l}\text { PhD-SNP } \\
\text { Expected } \\
\text { Accuracy }\end{array}$ & $\begin{array}{l}\text { SNAP } \\
\text { Prediction }\end{array}$ & $\begin{array}{l}\text { SNAP } \\
\text { Expected } \\
\text { Accuracy }\end{array}$ \\
\hline S2P & D $^{\mathrm{a}}$ & 0.549464 & $\mathrm{D}$ & 0.856822 & $\mathrm{~N}$ & 0.832104 & $\mathrm{~N}^{\mathrm{b}}$ & 0.768234 \\
\hline R9C & $\mathrm{N}$ & 0.738345 & $\mathrm{D}$ & 0.409295 & $\mathrm{~N}$ & 0.718714 & $\mathrm{~N}$ & 0.611679 \\
\hline R19H & $\mathrm{D}$ & 0.755662 & $\mathrm{D}$ & 0.765367 & $\mathrm{~N}$ & 0.552027 & $\mathrm{D}$ & 0.885196 \\
\hline R19C & $\mathrm{D}$ & 0.869084 & $\mathrm{D}$ & 0.913793 & $\mathrm{D}$ & 0.607981 & $\mathrm{D}$ & 0.869565 \\
\hline R20H & $\mathrm{N}$ & 0.602564 & $\mathrm{~N}$ & 0.742731 & $\mathrm{~N}$ & 0.832104 & $\mathrm{D}$ & 0.555519 \\
\hline G23S & $\mathrm{D}$ & 0.755662 & $\mathrm{D}$ & 0.571214 & $\mathrm{~N}$ & 0.552027 & $\mathrm{D}$ & 0.720388 \\
\hline
\end{tabular}


bioRxiv preprint doi: https://doi.org/10.1101/240820; this version posted January 3,2018 . The copyright holder for this preprint (which was not certified by peer review) is the author/funder, who has granted bioRxiv a license to display the preprint in perpetuity. It is made available under aCC-BY-NC-ND 4.0 International license.

\begin{tabular}{|c|c|c|c|c|c|c|c|c|}
\hline G23D & $\mathrm{D}$ & 0.869084 & $\mathrm{D}$ & 0.877061 & $\mathrm{D}$ & 0.732603 & $\mathrm{D}$ & 0.885196 \\
\hline V25G & $\mathrm{D}$ & 0.869084 & $\mathrm{D}$ & 0.81934 & $\mathrm{D}$ & 0.773385 & $\mathrm{D}$ & 0.720388 \\
\hline $\mathrm{V} 25 \mathrm{~L}$ & $\mathrm{D}$ & 0.718713 & $\mathrm{D}$ & 0.771193 & $\mathrm{D}$ & 0.577953 & $\mathrm{D}$ & 0.555519 \\
\hline $\mathrm{E} 28 \mathrm{~K}$ & $\mathrm{D}$ & 0.755662 & $\mathrm{D}$ & 0.841829 & $\mathrm{~N}$ & 0.508246 & $\mathrm{D}$ & 0.622082 \\
\hline Q29R & $\mathrm{D}$ & 0.755662 & $\mathrm{D}$ & 0.877061 & $\mathrm{~N}$ & 0.508246 & $\mathrm{D}$ & 0.555519 \\
\hline Q29P & $\mathrm{D}$ & 0.869084 & $\mathrm{D}$ & 0.877061 & $\mathrm{D}$ & 0.773385 & $\mathrm{D}$ & 0.805103 \\
\hline $\mathrm{R} 32 \mathrm{C}$ & $\mathrm{D}$ & 0.654946 & $\mathrm{D}$ & 0.783358 & $\mathrm{~N}$ & 0.782877 & $\mathrm{D}$ & 0.720388 \\
\hline D33E & $\mathrm{N}$ & 0.631518 & $\mathrm{D}$ & 0.81934 & $\mathrm{~N}$ & 0.782877 & $\mathrm{~N}$ & 0.665242 \\
\hline $\mathrm{D} 33 \mathrm{H}$ & $\mathrm{D}$ & 0.755662 & $\mathrm{D}$ & 0.774944 & $\mathrm{~N}$ & 0.68184 & $\mathrm{D}$ & 0.720388 \\
\hline D33N & $\mathrm{D}$ & 0.718713 & $\mathrm{D}$ & 0.765367 & $\mathrm{~N}$ & 0.782877 & $\mathrm{D}$ & 0.622082 \\
\hline $\mathrm{A} 36 \mathrm{~V}$ & $\mathrm{~N}$ & 0.631518 & $\mathrm{D}$ & 0.783358 & $\mathrm{~N}$ & 0.782877 & $\mathrm{~N}$ & 0.611679 \\
\hline $\mathrm{A} 36 \mathrm{E}$ & $\mathrm{N}$ & 0.736888 & $\mathrm{D}$ & 0.587706 & $\mathrm{~N}$ & 0.782877 & $\mathrm{~N}$ & 0.708784 \\
\hline A39V & $\mathrm{N}$ & 0.628313 & $\mathrm{D}$ & 0.877061 & $\mathrm{~N}$ & 0.782877 & $\mathrm{~N}$ & 0.611679 \\
\hline $\mathrm{R} 46 \mathrm{C}$ & $\mathrm{N}$ & 0.625291 & $\mathrm{D}$ & 0.81934 & $\mathrm{~N}$ & 0.660879 & $\mathrm{~N}$ & 0.611679 \\
\hline R48Q & $\mathrm{D}$ & 0.605483 & $\mathrm{D}$ & 0.765367 & $\mathrm{~N}$ & 0.58231 & $\mathrm{D}$ & 0.805103 \\
\hline F51L & $\mathrm{D}$ & 0.869084 & $\mathrm{D}$ & 0.76087 & $\mathrm{D}$ & 0.732603 & $\mathrm{D}$ & 0.848454 \\
\hline D52E & $\mathrm{D}$ & 0.869084 & $\mathrm{D}$ & 0.81934 & $\mathrm{D}$ & 0.577953 & $\mathrm{D}$ & 0.848454 \\
\hline E56K & $\mathrm{D}$ & 0.869084 & $\mathrm{D}$ & 0.774944 & $\mathrm{D}$ & 0.67621 & $\mathrm{D}$ & 0.805103 \\
\hline T57I & $\mathrm{D}$ & 0.869084 & $\mathrm{D}$ & 0.877061 & $\mathrm{D}$ & 0.732603 & $\mathrm{D}$ & 0.555519 \\
\hline T57A & $\mathrm{D}$ & 0.605483 & $\mathrm{D}$ & 0.877061 & $\mathrm{D}$ & 0.607981 & $\mathrm{~N}$ & 0.500147 \\
\hline G61R & $\mathrm{D}$ & 0.869084 & $\mathrm{D}$ & 0.877061 & $\mathrm{D}$ & 0.607981 & $\mathrm{D}$ & 0.848454 \\
\hline G61D & $\mathrm{D}$ & 0.869084 & $\mathrm{D}$ & 0.877061 & $\mathrm{D}$ & 0.577953 & $\mathrm{D}$ & 0.720388 \\
\hline F63L & $\mathrm{D}$ & 0.505959 & $\mathrm{D}$ & 0.76087 & $\mathrm{D}$ & 0.607981 & $\mathrm{~N}$ & 0.583594 \\
\hline A64T & $\mathrm{N}$ & 0.736888 & $\mathrm{D}$ & 0.587706 & $\mathrm{~N}$ & 0.832104 & $\mathrm{~N}$ & 0.583594 \\
\hline $\mathrm{R} 67 \mathrm{H}$ & $\mathrm{D}$ & 0.654946 & $\mathrm{D}$ & 0.573143 & $\mathrm{~N}$ & 0.718714 & $\mathrm{D}$ & 0.622082 \\
\hline R67C & $\mathrm{D}$ & 0.718713 & $\mathrm{D}$ & 0.783358 & $\mathrm{~N}$ & 0.660879 & $\mathrm{D}$ & 0.720388 \\
\hline R67L & $\mathrm{N}$ & 0.712788 & $\mathrm{D}$ & 0.858321 & $\mathrm{~N}$ & 0.552027 & $\mathrm{~N}$ & 0.500147 \\
\hline R69W & $\mathrm{N}$ & 0.738345 & $\mathrm{D}$ & 0.461769 & $\mathrm{~N}$ & 0.552027 & $\mathrm{~N}$ & 0.500147 \\
\hline G70D & $\mathrm{N}$ & 0.631518 & $\mathrm{D}$ & 0.656672 & $\mathrm{D}$ & 0.588855 & $\mathrm{~N}$ & 0.583594 \\
\hline L71V & $\mathrm{N}$ & 0.736888 & $\mathrm{D}$ & 0.571214 & $\mathrm{~N}$ & 0.782877 & $\mathrm{~N}$ & 0.665242 \\
\hline K75Q & $\mathrm{N}$ & 0.738345 & $\mathrm{D}$ & 0.409295 & $\mathrm{~N}$ & 0.718714 & $\mathrm{~N}$ & 0.665242 \\
\hline $\mathrm{Y} 77 \mathrm{H}$ & $\mathrm{D}$ & 0.789035 & $\mathrm{D}$ & 0.766117 & $\mathrm{~N}$ & 0.446708 & $\mathrm{D}$ & 0.885196 \\
\hline $\mathrm{T} 80 \mathrm{M}$ & $\mathrm{N}$ & 0.631518 & $\mathrm{~N}$ & 0.65022 & $\mathrm{~N}$ & 0.782877 & $\mathrm{~N}$ & 0.500147 \\
\hline R83W & $\mathrm{N}$ & 0.625291 & $\mathrm{~N}$ & 0.642291 & $\mathrm{~N}$ & 0.58231 & $\mathrm{~N}$ & 0.500147 \\
\hline R86W & $\mathrm{N}$ & 0.75204 & $\mathrm{D}$ & 0.573143 & $\mathrm{~N}$ & 0.508246 & $\mathrm{~N}$ & 0.583594 \\
\hline S98L & $\mathrm{N}$ & 0.602564 & $\mathrm{D}$ & 0.913793 & $\mathrm{~N}$ & 0.68184 & $\mathrm{~N}$ & 0.500147 \\
\hline G104R & $\mathrm{N}$ & 0.733469 & $\mathrm{D}$ & 0.913793 & $\mathrm{~N}$ & 0.718714 & $\mathrm{~N}$ & 0.611679 \\
\hline D112E & $\mathrm{N}$ & 0.738345 & $\mathrm{D}$ & 0.508996 & $\mathrm{~N}$ & 0.832104 & $\mathrm{~N}$ & 0.768234 \\
\hline L113P & $\mathrm{D}$ & 0.505959 & $\mathrm{D}$ & 0.877061 & $\mathrm{D}$ & 0.67621 & $\mathrm{~N}$ & 0.665242 \\
\hline L115R & $\mathrm{N}$ & 0.625291 & $\mathrm{D}$ & 0.877061 & $\mathrm{D}$ & 0.607981 & $\mathrm{~N}$ & 0.554397 \\
\hline $\mathrm{C} 117 \mathrm{Y}$ & $\mathrm{D}$ & 0.869084 & $\mathrm{D}$ & 0.771193 & $\mathrm{D}$ & 0.577953 & $\mathrm{D}$ & 0.720388 \\
\hline
\end{tabular}


bioRxiv preprint doi: https://doi.org/10.1101/240820; this version posted January 3,2018 . The copyright holder for this preprint (which was not certified by peer review) is the author/funder, who has granted bioRxiv a license to display the preprint in perpetuity. It is made available under aCC-BY-NC-ND 4.0 International license.

\begin{tabular}{lllllllll}
\hline R122C & N & 0.602564 & $\mathrm{D}$ & 0.656672 & $\mathrm{~N}$ & 0.660879 & $\mathrm{D}$ & 0.555519 \\
\hline P134L & $\mathrm{N}$ & 0.602564 & $\mathrm{D}$ & 0.856822 & $\mathrm{~N}$ & 0.832104 & $\mathrm{~N}$ & 0.583594 \\
\hline Y151C & $\mathrm{D}$ & 0.869084 & $\mathrm{D}$ & 0.656672 & $\mathrm{D}$ & 0.67621 & $\mathrm{D}$ & 0.720388 \\
\hline R155C & $\mathrm{D}$ & 0.755662 & $\mathrm{D}$ & 0.856822 & $\mathrm{~N}$ & 0.552027 & $\mathrm{D}$ & 0.805103 \\
\hline R156W & $\mathrm{D}$ & 0.869084 & $\mathrm{D}$ & 0.771193 & $\mathrm{D}$ & 0.588855 & $\mathrm{D}$ & 0.848454 \\
\hline R156Q & $\mathrm{D}$ & 0.755662 & $\mathrm{D}$ & 0.765367 & $\mathrm{~N}$ & 0.660879 & $\mathrm{D}$ & 0.848454 \\
\hline
\end{tabular}

Table 2. Prediction of Disease Related Mutations by Predict-SNP, MAPP, PhD-SNP, and SNAP. ${ }^{\text {a }}$ Deleterious effect; ${ }^{\mathrm{b}}$ Neutral effect.

\subsection{I-mutant 3.0 and Mupro analysis of protein's stability change upon amino acid substitution}

Prediction of the changes in protein's stability upon amino acid substitution performed by I-Mutant 3.0 server and Mupro Server predicted 46 and 41 amino acid substitution, out of 53, respectively, to bring decrease in the stability of the cyclin dependent kinase 1 protein encoded by CDKN1A gene. All Scores from these servers are represented in

Table 3. Stability change in I-Mutant 3.0 was calculated at $25^{\circ} \mathrm{C}$ and $\mathrm{pH} 7$ and expressed as DDG value=DG (new protein)- DG (wild type) in $\mathrm{kcal} / \mathrm{mol}$.

\begin{tabular}{|c|c|c|c|c|c|c|c|}
\hline SNP ID & Protein Id & Variant & $\begin{array}{l}\text { Sign of } \\
\text { DDG }\end{array}$ & $\begin{array}{l}\text { DDG value prediction } \\
\text { kcal/mol }\end{array}$ & $\mathbf{R I}$ & $\begin{array}{l}\text { MUpro } \\
\text { Prediction }\end{array}$ & $\begin{array}{l}\text { Confidence } \\
\text { score }\end{array}$ \\
\hline rs148679597 & ENSP00000244741 & $\mathrm{C} 117 \mathrm{Y}$ & $\mathrm{D}^{\mathrm{a}}$ & -0.34 & 0 & $\mathrm{I}^{\mathrm{b}}$ & 0.467551 \\
\hline rs4986867 & ENSP00000244741 & F63L & $\mathrm{D}$ & -0.99 & 7 & I & 0.69283285 \\
\hline rs12721594 & ENST00000244741 & R48Q & $\mathrm{D}$ & -0.79 & 8 & $\mathrm{D}$ & -0.96731 \\
\hline \multirow[t]{2}{*}{ rs45548832 } & ENSP00000244741 & $\mathrm{R} 67 \mathrm{H}$ & $\mathrm{D}$ & -1.19 & 9 & $\mathrm{D}$ & -1 \\
\hline & ENST00000244741 & R67L & $\mathrm{D}$ & -0.43 & 9 & $\mathrm{D}$ & -0.49746 \\
\hline rs114149607 & ENSP00000244741 & $\mathrm{T} 80 \mathrm{M}$ & $\mathrm{D}$ & -0.38 & 4 & I & 0.075972 \\
\hline rs 151314631 & ENSP00000244741 & $\mathrm{V} 25 \mathrm{~L}$ & $\mathrm{D}$ & -1 & 7 & $\mathrm{D}$ & -0.27178 \\
\hline rs199801759 & ENSP00000244741 & Y151C & $\mathrm{D}$ & -1.33 & 0 & $\mathrm{D}$ & -1 \\
\hline rs368128681 & ENSP00000244741 & G23D & $\mathrm{D}$ & -0.93 & 6 & $\mathrm{D}$ & -0.43437301 \\
\hline rs369108178 & ENSP00000244741 & P134L & I & -0.12 & 2 & I & 0.491005 \\
\hline rs370291979 & ENSP00000244741 & T57A & $\mathrm{D}$ & -0.93 & 7 & $\mathrm{D}$ & -0.68358 \\
\hline
\end{tabular}


bioRxiv preprint doi: https://doi.org/10.1101/240820; this version posted January 3, 2018. The copyright holder for this preprint (which was not certified by peer review) is the author/funder, who has granted bioRxiv a license to display the preprint in perpetuity. It is made available under aCC-BY-NC-ND 4.0 International license.

\begin{tabular}{|c|c|c|c|c|c|c|c|}
\hline rs371219528 & ENSP00000244741 & $\mathrm{S} 2 \mathrm{P}$ & I & -0.15 & 3 & I & 0.048219 \\
\hline rs372390764 & ENSP00000244741 & $\mathrm{R} 67 \mathrm{C}$ & $\mathrm{D}$ & -0.93 & 7 & $\mathrm{D}$ & -1 \\
\hline rs372882329 & ENSP00000244741 & L113P & $\mathrm{D}$ & -1.58 & 3 & $\mathrm{D}$ & -0.76282 \\
\hline rs373450720 & ENSP00000244741 & $\mathrm{R} 32 \mathrm{C}$ & $\mathrm{D}$ & -0.79 & 5 & $\mathrm{D}$ & -1 \\
\hline rs374154006 & ENSP00000244741 & R156W & $\mathrm{D}$ & -0.61 & 5 & $\mathrm{D}$ & -0.31493523 \\
\hline rs374965936 & ENSP00000244741 & $\mathrm{R} 83 \mathrm{~W}$ & $\mathrm{D}$ & -0.26 & 3 & I & 0.46858235 \\
\hline rs375050346 & ENSP00000244741 & $\mathrm{R} 20 \mathrm{H}$ & $\mathrm{D}$ & -1.42 & 9 & $\mathrm{D}$ & -0.86875024 \\
\hline \multirow[t]{2}{*}{ rs376481017 } & ENSP00000244741 & D33N & $\mathrm{D}$ & -0.73 & 0 & $\mathrm{D}$ & -0.88042 \\
\hline & ENSP00000244741 & D33H & $\mathrm{D}$ & -0.52 & 3 & $\mathrm{D}$ & -1 \\
\hline rs541505866 & ENSP00000244741 & D52E & I & 0.14 & 6 & $\mathrm{D}$ & -1 \\
\hline rs559151286 & ENSP00000244741 & $\mathrm{A} 39 \mathrm{~V}$ & $\mathrm{D}$ & -0.25 & 4 & I & 0.959321 \\
\hline rs569995916 & ENSP00000244741 & G104R & I & -0.27 & 1 & I & 0.66691507 \\
\hline \multirow[t]{2}{*}{ rs746709171 } & ENSP00000244741 & $\mathrm{A} 36 \mathrm{E}$ & $\mathrm{D}$ & -0.34 & 6 & $\mathrm{D}$ & -0.19889 \\
\hline & ENSP00000244741 & $\mathrm{A} 36 \mathrm{~V}$ & $\mathrm{D}$ & -0.15 & 4 & $\mathrm{D}$ & -0.1802 \\
\hline rs748758330 & ENSP00000244741 & R69W & $\mathrm{D}$ & -0.3 & 7 & I & 0.050719 \\
\hline rs748842488 & ENSP00000244741 & F51L & $\mathrm{D}$ & -0.89 & 3 & $\mathrm{D}$ & -0.68682 \\
\hline rs750249677 & ENSP00000244741 & $\mathrm{R} 155 \mathrm{C}$ & $\mathrm{D}$ & -1.23 & 4 & $\mathrm{D}$ & -0.73539 \\
\hline rs752126483 & ENSP00000244741 & D112E & I & 0.3 & 8 & $\mathrm{D}$ & -0.58613 \\
\hline rs752557277 & ENSP00000244741 & $\mathrm{R} 9 \mathrm{C}$ & $\mathrm{D}$ & -0.8 & 3 & $\mathrm{D}$ & -0.80301 \\
\hline rs752792828 & ENSP00000244741 & $\mathrm{Y} 77 \mathrm{H}$ & $\mathrm{D}$ & -1.08 & 7 & $\mathrm{D}$ & -1 \\
\hline rs753291170 & ENSP00000244741 & E56K & $\mathrm{D}$ & -0.82 & 9 & $\mathrm{D}$ & -0.25532 \\
\hline
\end{tabular}


bioRxiv preprint doi: https://doi.org/10.1101/240820; this version posted January 3, 2018. The copyright holder for this preprint (which was not certified by peer review) is the author/funder, who has granted bioRxiv a license to display the preprint in perpetuity. It is made available under aCC-BY-NC-ND 4.0 International license.

\begin{tabular}{|c|c|c|c|c|c|c|c|}
\hline rs753529000 & ENSP00000244741 & $\mathrm{R} 122 \mathrm{C}$ & $\mathrm{D}$ & -0.85 & 2 & $\mathrm{D}$ & -0.04479 \\
\hline \multirow[t]{2}{*}{ rs 756383281} & ENSP00000244741 & Q29P & $\mathrm{D}$ & -0.65 & 4 & $\mathrm{D}$ & -0.70387 \\
\hline & ENSP00000244741 & Q29R & $\mathrm{D}$ & -0.31 & 1 & $\mathrm{D}$ & -0.28217 \\
\hline rs758877354 & ENSP00000244741 & G61R & $\mathrm{D}$ & -0.4 & 3 & $\mathrm{D}$ & -0.75934 \\
\hline rs760466052 & ENSP00000244741 & S98L & I & 0.34 & 1 & I & 1 \\
\hline rs762978904 & ENSP00000244741 & $\mathrm{R} 46 \mathrm{C}$ & $\mathrm{D}$ & -0.88 & 5 & $\mathrm{D}$ & -0.93791 \\
\hline rs763967941 & ENSP00000244741 & E28K & $\mathrm{D}$ & -1.22 & 9 & I & 0.377192 \\
\hline rs765248879 & ENSP00000244741 & V25G & $\mathrm{D}$ & -2.43 & 9 & $\mathrm{D}$ & -1 \\
\hline rs765503766 & ENSP00000244741 & D33E & I & -0.01 & 6 & $\mathrm{D}$ & -0.59711968 \\
\hline rs767974221 & ENSP00000244741 & L115R & $\mathrm{D}$ & -1.72 & 7 & $\mathrm{D}$ & -1 \\
\hline rs769049147 & ENSP00000244741 & G70D & $\mathrm{D}$ & -0.86 & 5 & $\mathrm{D}$ & -0.21741 \\
\hline rs769229553 & ENSP00000244741 & $\mathrm{R} 19 \mathrm{C}$ & $\mathrm{D}$ & -1.06 & 5 & $\mathrm{D}$ & -0.34018 \\
\hline rs770464093 & ENSP00000244741 & G23S & $\mathrm{D}$ & -1.26 & 8 & $\mathrm{D}$ & -1 \\
\hline rs771753909 & ENSP00000244741 & L71V & $\mathrm{D}$ & -1.55 & 8 & $\mathrm{D}$ & -0.63308 \\
\hline rs772419969 & ENSP00000244741 & T57I & $\mathrm{D}$ & -0.21 & 0 & $\mathrm{D}$ & -0.85972 \\
\hline rs778199042 & ENSP00000244741 & G61D & $\mathrm{D}$ & -1.03 & 7 & $\mathrm{D}$ & -0.50922 \\
\hline rs779063779 & ENSP00000244741 & $\mathrm{R} 156 \mathrm{Q}$ & $\mathrm{D}$ & -1.27 & 9 & $\mathrm{D}$ & -0.74046 \\
\hline rs779250316 & ENSP00000244741 & $\mathrm{R} 19 \mathrm{H}$ & $\mathrm{D}$ & -1.42 & 9 & I & 0.31757804 \\
\hline rs779717089 & ENSP00000244741 & R86W & $\mathrm{D}$ & -0.3 & 2 & $\mathrm{D}$ & -0.49632 \\
\hline rs780909704 & ENSP00000244741 & A64T & $\mathrm{D}$ & -0.67 & 6 & $\mathrm{D}$ & -0.07652 \\
\hline rs935843132 & ENSP00000244741 & K75Q & $\mathrm{D}$ & -0.27 & 1 & $\mathrm{D}$ & -0.20877166 \\
\hline
\end{tabular}




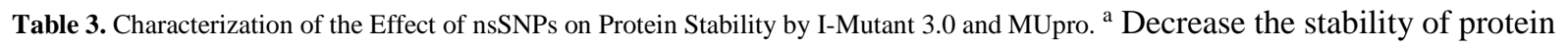
structure; ${ }^{\mathrm{b}}$ Increase the stability of protein structure.

\subsection{Screening of most frequent deleterious amino acid substitution}

Following the screening of harmful nsSNPs by SIFT and Polyphen-2 server, the rest 53 nsSNPs, predicted to be deleterious, were further analyzed. A distribution of harmful substitution predicted by Predict SNP, MAPP, PhD-SNP, SNAP, I-Mutant 3.0 and Mupro is shown in Figure 1. Disease related mutations exposed by Predict SNP, MAPP, PhD-SNP and SNAP predicted 58.5\%, 93.3\%, 35.8\%, and 54.7\% nsSNPs, respectively, as deleterious. Analysis by I-Mutant 3.0 and Mupro suggested that $86.8 \%$ and $77.4 \%$ nsSNPs, respectively, were affected by amino acid substitution and resulted in decrease in stability. Frequency of deleterious nsSNPs in various servers was calculated by IBM SPSS v20 and Microsoft office 2013.

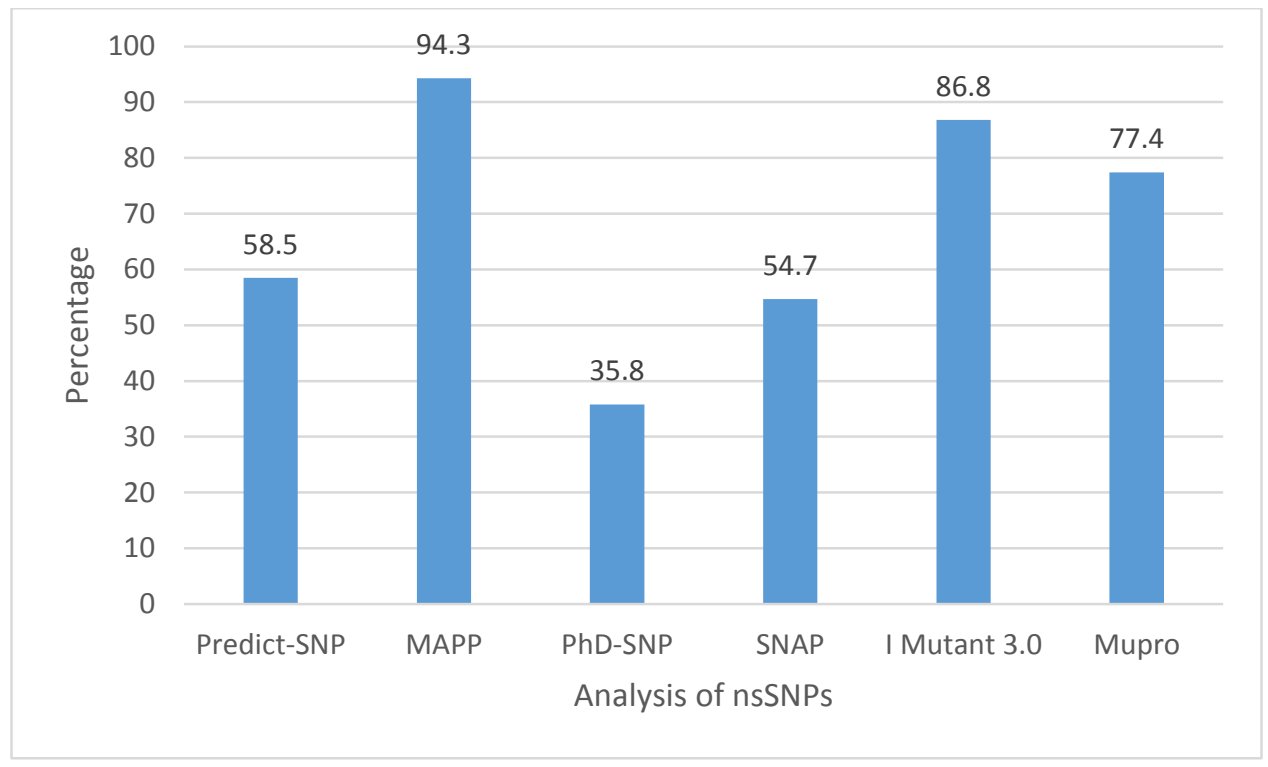

Figure 1. Overview of the analysis of deleterious and stability decreased cyclin dependent kinase inhibitor 1 due to nsSNPs by various in silico tools

Prediction and distribution of deleterious nsSNPs by all six methods- Predict-SNP, MAPP, PhD-SNP, SNAP, I-Mutant 3.0 and Mupro, illustrated in Figure 2, revealed the most frequent 12 amino acid substitution predicted to be either "Deleterious" or "Stability Decreasing". Amino acid substitution- R19C, G23D, V25G, V25L, Q29P, F51L, E56K, T57I, G61R, G61D, Y151C and R156W were predicted as most harmful by all the above methods. 
bioRxiv preprint doi: https://doi.org/10.1101/240820; this version posted January 3, 2018. The copyright holder for this preprint (which was not certified by peer review) is the author/funder, who has granted bioRxiv a license to display the preprint in perpetuity. It is made available under aCC-BY-NC-ND 4.0 International license.

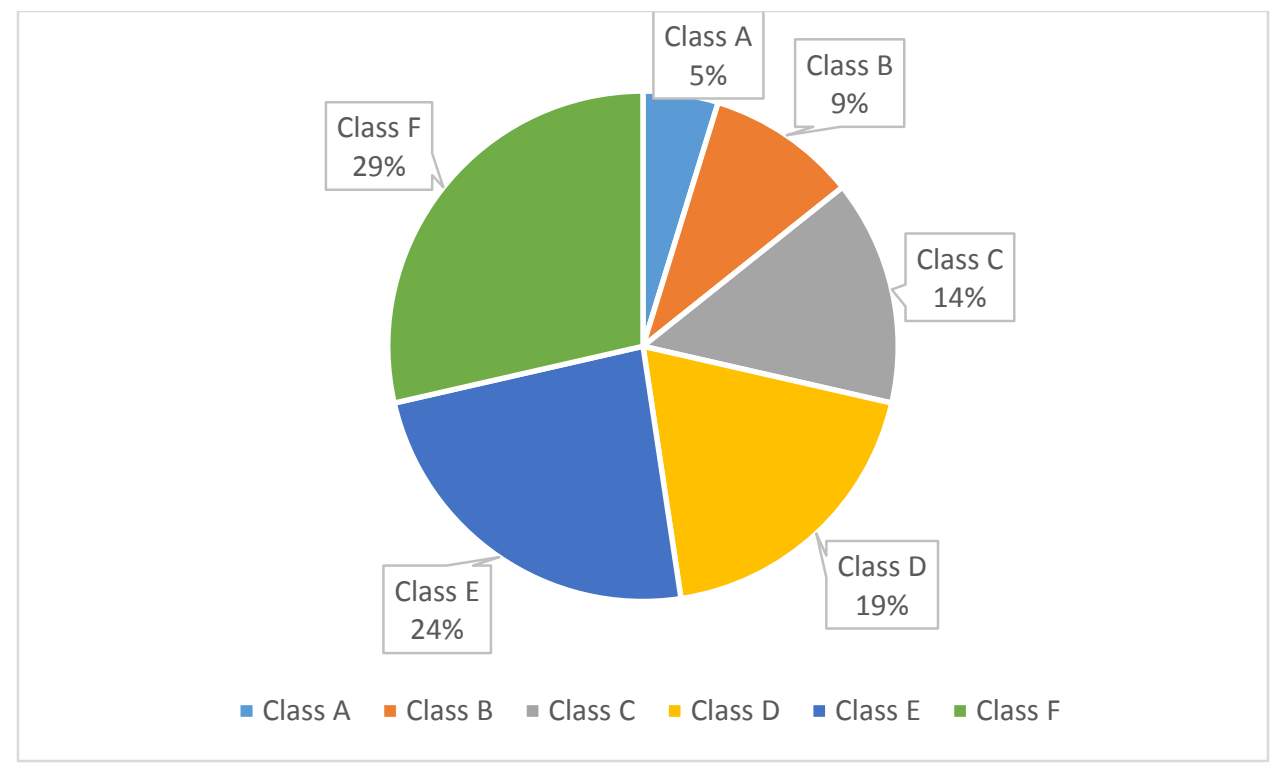

Figure 2. Prediction and distribution of deleterious nsSNPs by Predict-SNP, MAPP, PhD-SNP, SNAP, I-Mutant 3.0 and Mupro methods; Class A, predicted damaging by at least one method, T80M, R83W, S98L, G104R and P134L; Class B, predicted damaging by at least two methods, D33E,A39V, R69W, D112E; Class C, predicted damaging by at least three methods, R9C, R20H, A36V, A36E, R46C, A64T, R67L, L71V, R86W; Class D, predicted damaging by at least four methods, S2P, R19H, E28K, F63L, G70D, K75Q, L115R, R122C; Class E, predicted damaging by at least five methods, G23S, Q29R, R32C, D33H, D33N, R48Q, D52E, T57A, R67H, R67C, Y77H, L113P, C117Y, R155C, R156Q; Class F, predicted damaging by all six methods, R19C, G23D, V25G, V25L, Q29P, F51L, E56K, T57I, G61R, G61D, Y151C, R156W.

\subsection{Structural conformation and conservation analysis by ConSurf sever}

A colorimetric conservation score was produced as result by the ConSurf server (Figure 3). Highly conserved functional region of the protein was revealed by ConSurf tool. It was found that R19C, G23D, F51L, Y151C and R156W have a conservation score of 9; V25G, V25L, Q29P, T57I, G61R and G61D have a conservation score of 8; E56K have a conservation score of 7. 


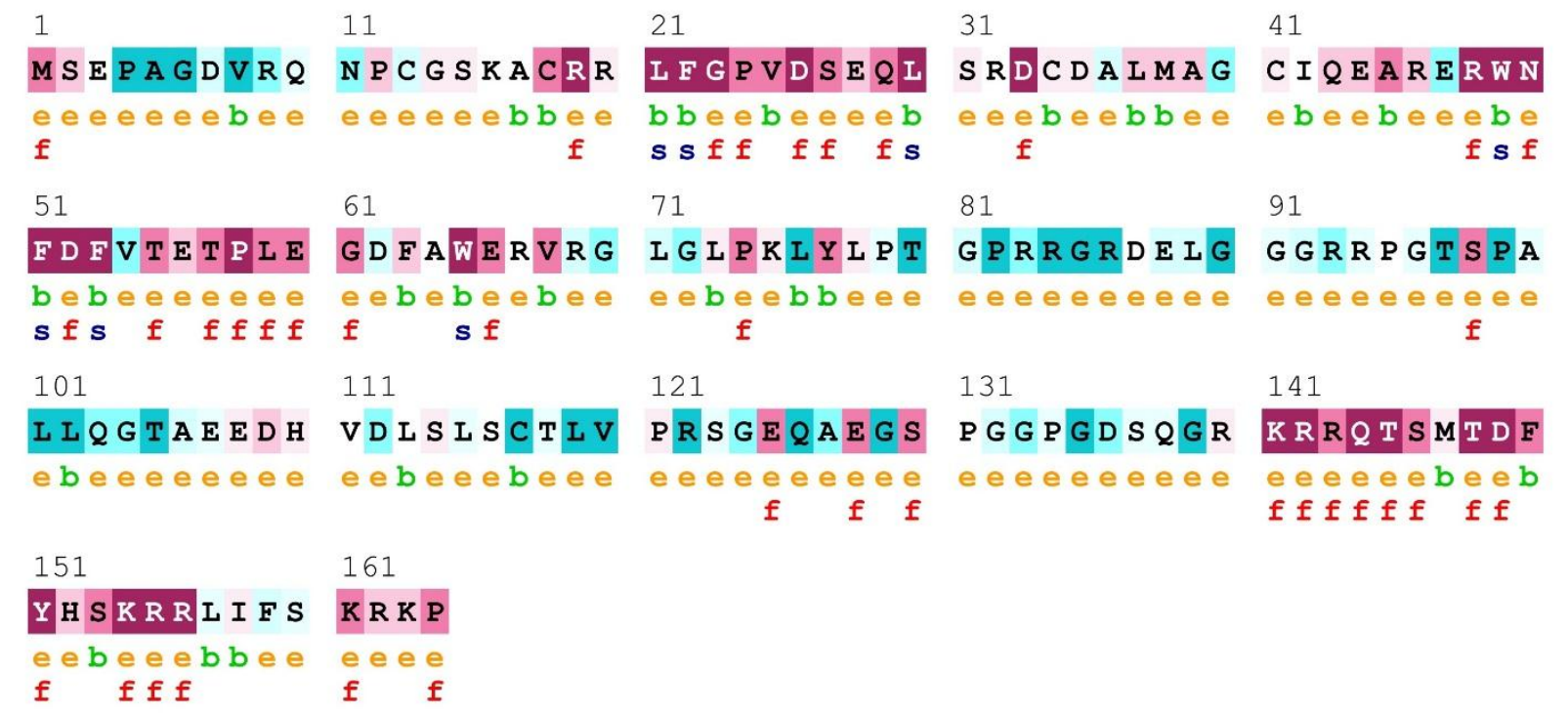

The conservation scale:

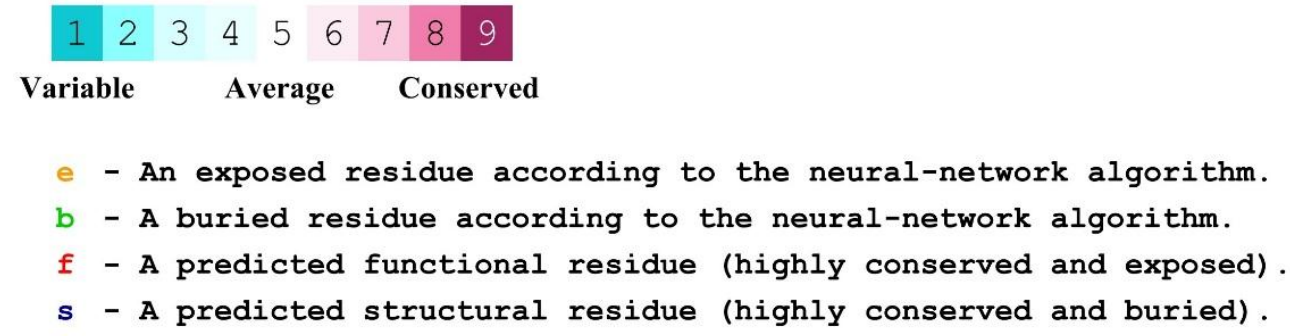

Figure 3. Prediction of evolutionary conserved amino acid residues by ConSurf server. Conservation score is represented as the color coding bars.

\subsection{Prediction of secondary structure by PSIPRED}

Distribution of alpha helix and beta sheet and coil is exposed by PSIPRED (Figure 4). Among the secondary structures, the highest in percentage was coils (82.93\%) followed by alpha helix (14.02\%) and beta sheet (3.05\%). 


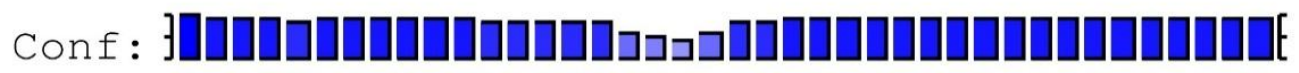

Pred:

Pred: CCCCCCCCCCCCCCCCCCCCCCCCCCHHHHHHHHHHHHHH

AA: MSEPAGDVRQNPCGSKACRRLFGPVDSEQL,SRDCDALMAG

$\begin{array}{llll}10 & 20 & 30 & 40\end{array}$

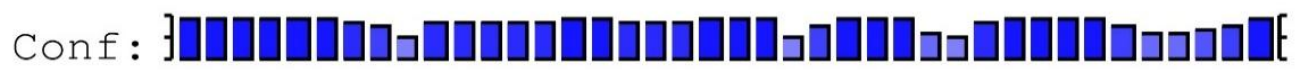

Pred: $\longrightarrow \longrightarrow$

Pred: HHHHHHHHHCCCCCCCCCCCCCCEEEEECCCCCCCCCCCC

AA: CIQEARERWNFDFVTETPLẸGDFAWERVRGLGLPKLYLPT

$\begin{array}{llll}1 & 1 & 1 & 1 \\ 50 & 60 & 70\end{array}$

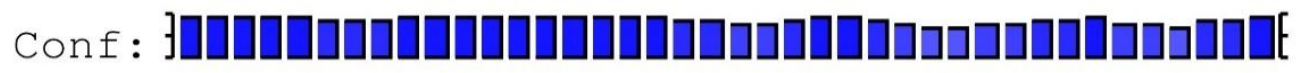

Pred:

Pred: CCCCCCCCCCCCCCCCCCCCCCCCCCCCCCCCCCCCCCCC

AA: GPRRGRDELGGGRRPGTSPALLQGTAEEDHVDLSLSCTLV

$\begin{array}{llll}90 & 100 & 110 & 120\end{array}$

Conf : J

Pred:

Pred: CCCCCCCCCCCCCCCCCCCCCCCCCCCCCCCCCCCCCCCC

AA: PRSGEQAEGSPGGPGDSQGRKRRQTSMTDFYHSKRRLIFS

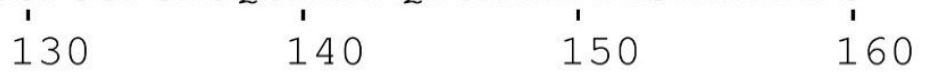

Conf : ][⿴囗十

Pred:

Pred: CCCC

AA: KRKP

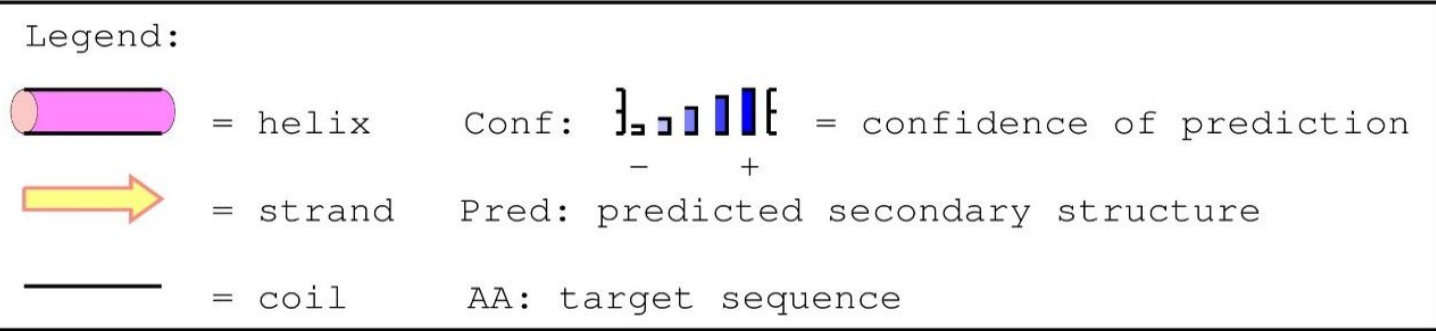

Figure 4. Prediction of secondary structure of by PSIPRED server 
bioRxiv preprint doi: https://doi.org/10.1101/240820; this version posted January 3, 2018. The copyright holder for this preprint (which was not certified by peer review) is the author/funder, who has granted bioRxiv a license to display the preprint in perpetuity. It is made available under aCC-BY-NC-ND 4.0 International license.

\subsection{Homology Modelling}

Structure refined and energy minimized homology models of CDKN1A by MUSTER, Phyre-2, RaptorX and Swiss model serves are illustrated using computational program Discovery Studio Modeling Environment 2016 (Figure 5). These models were checked for validation through Ramachandran plot analysis and QMEAN Z scores.

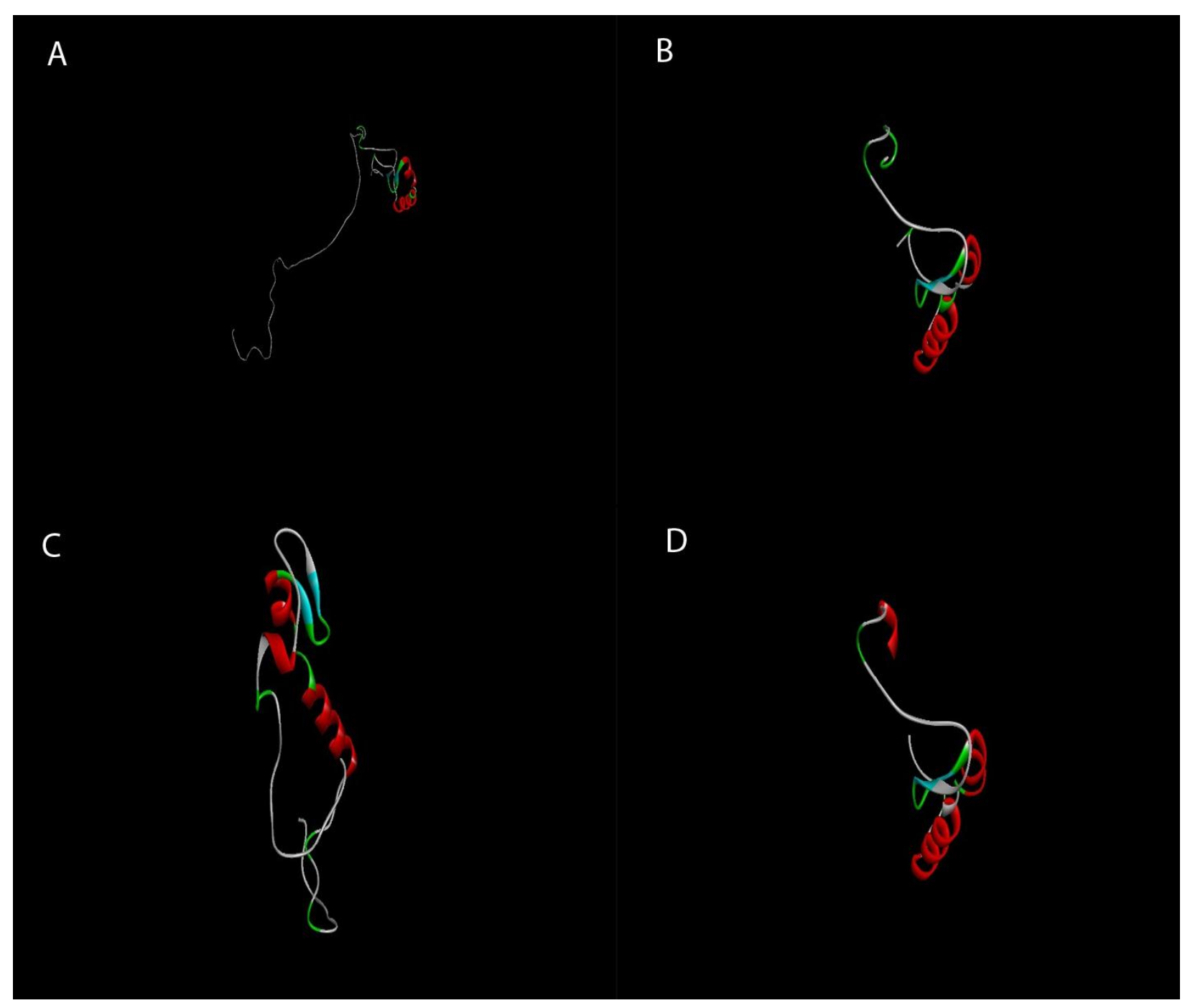

Figure 5. Homology models from different servers; A. CDKN1A_Muster, B. CDKN1A_Phyre2, C. CDKN1A_RaptorX, D. CDKN1A_Swiss model

\subsubsection{Ramachandran plot analysis}

Ramachandran plot is an $\mathrm{x}-\mathrm{y}$ plot of phi/psi dihedral angles between NC-alpha and Calpha-C bonds to evaluate a protein's backbone conformation. Ramachandran plots of structure refined and energy minimized homology models are illustrated in Figure 6. 


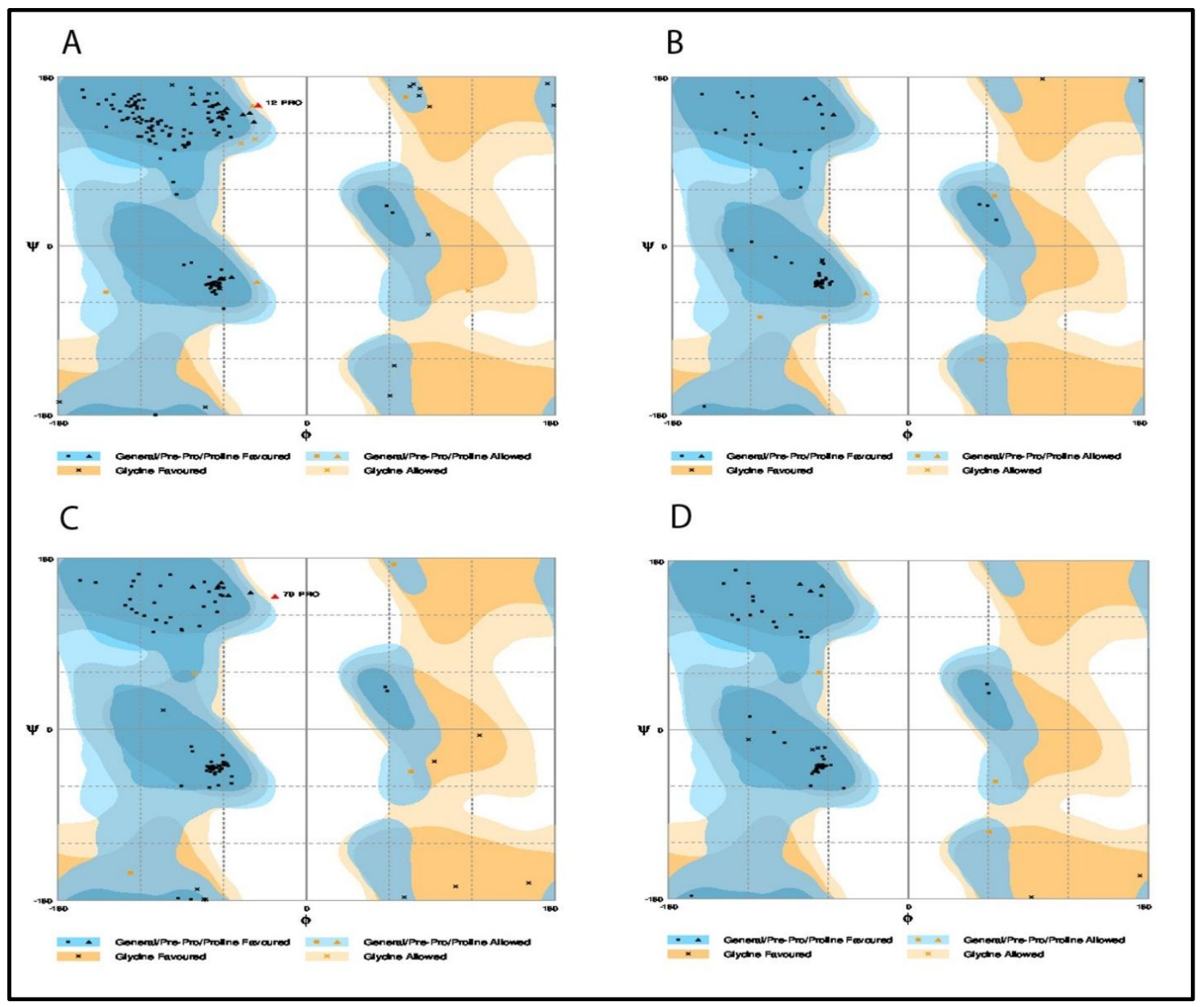

Figure 6. Ramachandran plots of different models; A. CDKN1A_Muster, B. CDKN1A_Phyre2, C. CDKN1A_RaptorX, D. CDKN1A_Swiss model

Table 4 represents the number of residues of different models (structure refined and energy minimized) in favored, allowed and outlier regions generated by the Ramachandran plot. Model generated by MUSTER had highest number of residues (95.1\%) in favored region followed by Swiss model (95\%), RaptorX (94.5\%) and Phyre-2 (92.3\%) server.

\begin{tabular}{lllllll|}
\hline \multirow{2}{*}{ Model } & \multicolumn{2}{l}{ Residues in favored region } & \multicolumn{2}{l}{ Residues in allowed region } & \multicolumn{2}{l|}{ Residues in outlier region } \\
\cline { 2 - 7 } & No of residues & $\%$ of residues & No of residues & \% of residues & No of residues & $\%$ of residues \\
\hline CDKN1A_muster & 154 & 95.1 & 7 & 4.3 & 1 & 0.6 \\
\hline CDKN1A_phyre 2 & 60 & 92.3 & 5 & 7.7 & 0 & 0 \\
\hline CDKN1A_raptor X & 86 & 94.5 & 4 & 4.4 & 1 & 1.1 \\
\hline CDKN1A_swiss model & 57 & 95.0 & 3 & 5.0 & 0 & 0 \\
\hline
\end{tabular}

Table 4. Ramachandran plot analysis of different homology models of CDKN1A

\subsubsection{QMEAN6 Z-value}


Table 5 shows the $Z$ scores obtained from QMEAN Server (Swiss Model) using structure refined and energy minimized models. Overall, the total QMEAN score ( $\mathrm{Z}$ value) of the models were better for RaptorX (-1.68), followed by Swiss model (-2.01), Phyre-2 (-2.12) and MUSTER (-2.30).

\begin{tabular}{lllllllll|}
\hline \multirow{2}{*}{ Models } & $\begin{array}{l}\text { All atom } \\
\text { pair } \\
\text { energy }\end{array}$ & $\begin{array}{l}\text { W } \\
\text { interaction } \\
\text { energy }\end{array}$ & $\begin{array}{l}\text { Solvation } \\
\text { energy }\end{array}$ & $\begin{array}{l}\text { Torsion } \\
\text { angle } \\
\text { energy }\end{array}$ & $\begin{array}{l}\text { Secondary } \\
\text { Structure } \\
\text { agreement }\end{array}$ & $\begin{array}{l}\text { ACC } \\
\text { Agreement }\end{array}$ & $\begin{array}{l}\text { Total } \\
\text { QMEAN } \\
\text { score }\end{array}$ \\
\hline CDKN1A_muster & -1.71 & -0.61 & -1.77 & -1.48 & -1.50 & -0.18 & -2.30 \\
\hline CDKN1A_phyre 2 & 0.62 & -1.22 & -1.55 & -0.54 & -1.83 & -1.13 & -2.12 \\
\hline $\begin{array}{l}\text { CDKN1A_raptor } \\
\text { X }\end{array}$ & -0.29 & -1.14 & -1.06 & -0.94 & -1.19 & 0.20 & -1.68 \\
\hline $\begin{array}{l}\text { CDKN1A_swiss } \\
\text { model }\end{array}$ & 0.53 & 0.05 & -1.35 & -1.02 & -1.57 & -1.36 & -2.01 \\
\hline
\end{tabular}

Table 5. Z scores obtained from QMEAN Server using energy minimized models

\subsection{3. $\mathrm{Z}$ scores of MUSTER generated models}

Ten corresponding template was constructed by MUSTER, but only one template was considered as good ( $\mathrm{Z}$ score >7.5). The rest had a $\mathrm{Z}$ score $<7.5$, thus was considered as bad (

\section{Table 6).}

\begin{tabular}{|c|c|c|c|c|c|c|}
\hline Rank & Template & Align length & Coverage & Z-score & $\overline{\text { Seq_id }}$ & Type \\
\hline 1 & $1 \mathrm{jsuC}$ & 69 & 0.420 & 9.345 & 0.391 & Good \\
\hline 2 & $3 \mathrm{jbmA}$ & 153 & 0.932 & 5.296 & 0.124 & Bad \\
\hline 3 & $2 \mathrm{mxxA}$ & 155 & 0.945 & 4.759 & 0.116 & $\mathrm{Bad}$ \\
\hline 4 & 2 muxA & 137 & 0.835 & 4.705 & 0.095 & $\mathrm{Bad}$ \\
\hline 5 & $5 \mathrm{n} 5 \mathrm{xA} 2$ & 162 & 0.987 & 4.623 & 0.111 & $\mathrm{Bad}$ \\
\hline 6 & $418 \mathrm{nA2}$ & 160 & 0.975 & 4.564 & 0.106 & Bad \\
\hline 7 & 4 fmyA & 162 & 0.987 & 4.426 & 0.086 & Bad \\
\hline 8 & 1su3B3 & 147 & 0.896 & 4.424 & 0.095 & $\mathrm{Bad}$ \\
\hline 9 & 4uisA1 & 140 & 0.853 & 4.331 & 0.093 & Bad \\
\hline 10 & $3 \mathrm{jb} 8 \mathrm{~A}$ & 161 & 0.981 & 4.318 & 0.112 & $\mathrm{Bad}$ \\
\hline
\end{tabular}

Table 6. Z scores of different models generated by MUSTER

\subsection{Visualization of selected mutations using Mutation 3D server}

Mutation 3D server illustrated selected harmful substitutions (Figure 7). Only one domain, CDI (PF02234), of the 164 amino acids in long cyclin dependent kinase inhibitor 1 protein (encoded by CDKN1A gene), was revealed. Among 12 mutations, 3 mutations were uncovered (R19C, Y151C, R156W), the rest 9 mutations (G23D, V25G, V25L, Q29P, F51L, E56K, T57I, G61R, and G61D) were in the domain region, thus considered as higher risk mutation for the cyclin dependent kinase inhibitor 1 protein. 


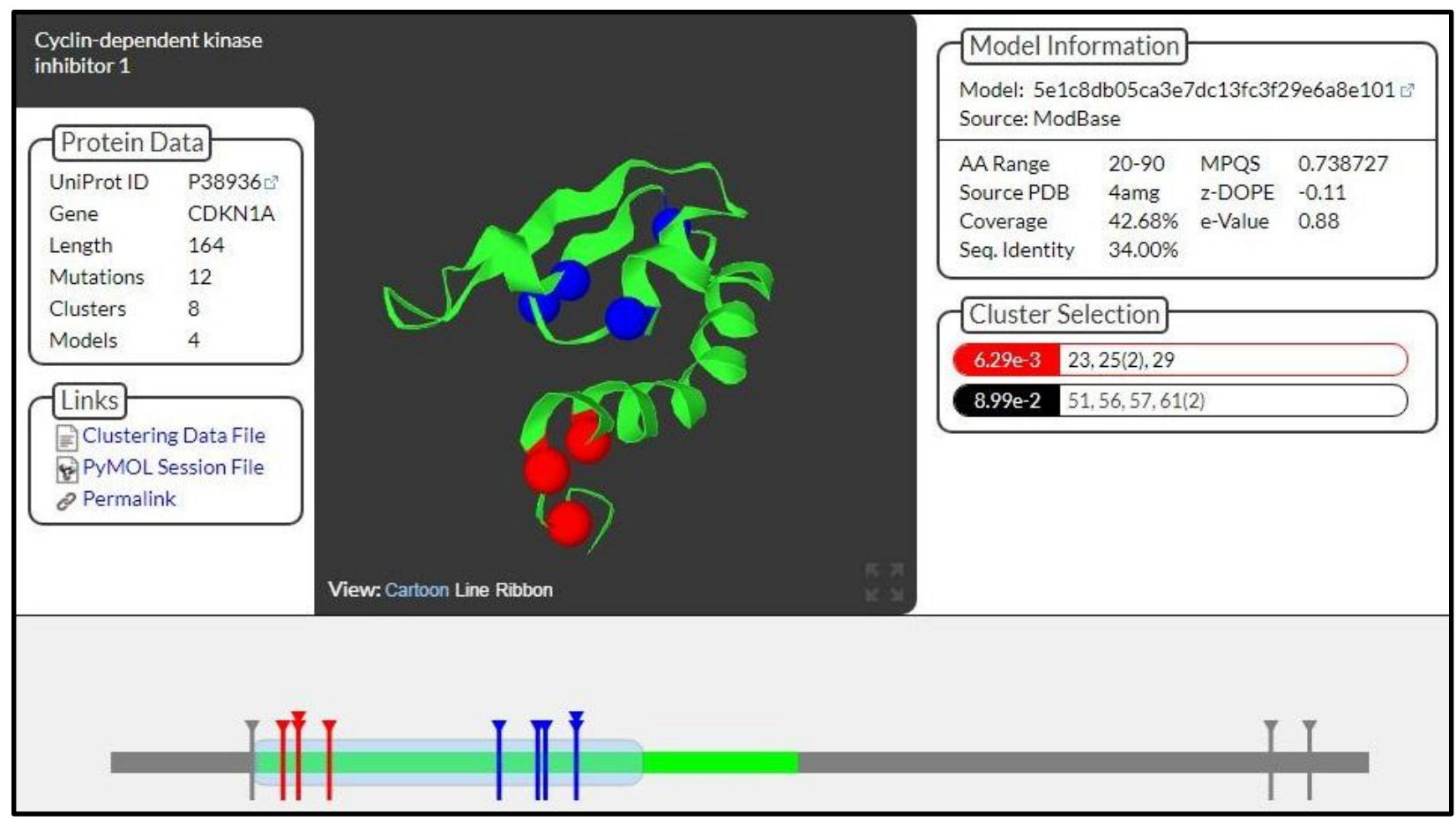

Figure 7. 3D structure of cyclin dependent kinase inhibitor 1 protein, generated by mutation 3D server, and representation of selected mutations on protein's domain

\subsection{Comparison of total energy and electrostatic constraints between energy minimized native and mutated models}

Total energy and electrostatic constraint (expresses as $\mathrm{kJ} / \mathrm{mol}$ ), after energy minimization by GROMOS 96 implementation of Swiss-PdbViewer, of the native (Swiss Model) structure and the mutant modeled structures are shown in Table 7. Out of 12 amino acid substitutions, G23D, V25L, F51L, E56K, T57I, G61R, G61D were found to have decrease in both total energy and electrostatic constraint in comparison with the native structure.

\begin{tabular}{lll}
\hline Amino acid variants & Total energy after minimization $\mathbf{( k J / m o l )}$ & Electrostatic constraint $(\mathbf{k J} / \mathbf{m o l})$ \\
\hline Native & -2257.007 & -1918.52 \\
\hline R19C & -2232.437 & -1700.97 \\
\hline G23D & -2502.866 & -2030.48 \\
\hline V25G & -2411.375 & -1883.49 \\
\hline V25L & -2473.934 & -1956.50 \\
\hline Q29P & -2278.592 & -1798.33 \\
\hline F51L & -2449.725 & -1954.00 \\
\hline E56K & -2491.817 & -1951.96 \\
\hline T57I & -2489.148 & -1963.93 \\
\hline G61R & -2813.512 & -2290.11 \\
\hline G61D & -2542.411 & -2025.01 \\
\hline
\end{tabular}

Table 7. Total energy and electrostatic constraint after energy minimization in native and mutated models 


\subsection{Biophysical analysis of selected amino acid substitutions}

Functional effect of missense amino acid substitution, analyzed by Align GVGD, is represented in

Table 8. All 12 nsSNPs existed at strongly conserved residues (GV=0). Nine variants, R19C, G23D, V25G, Q29P, T57I, G61R, G61D, Y151C, R156W, were predicted to be in the class C65. Thus, they were inferred as most likely to interfere with the function. One variant, E56K, was predicted to be in class C55. Thus, it was defined as interfering with function. The additional variants, F51L and V25L, were predicted as less likely to interfere with the function.

\begin{tabular}{llll}
\hline Amino acid variants & A-GVDV & & \\
\hline & GV & GD & Prediction class \\
\hline R19C & 0.00 & 179.53 & Class C65 \\
\hline G23D & 0.00 & 93.77 & Class C65 \\
\hline V25G & 108.79 & Class C65 \\
\hline V25L & 0.00 & 31.78 & Class C25 \\
\hline Q29P & 0.00 & 75.14 & Class C65 \\
\hline F51L & 0.00 & 21.82 & Class C15 \\
\hline E56K & 0.00 & 56.87 & Class C55 \\
\hline T57I & 0.00 & 89.28 & Class C65 \\
\hline G61R & 0.00 & 125.13 & Class C65 \\
\hline G61D & 0.00 & 93.77 & Class C65 \\
\hline R156W & 0.00 & 101.29 & Class C65 \\
\hline Y151C & 193.72 & Class C65 \\
\hline
\end{tabular}

Table 8. Analysis of the functional effect upon nonsynonymous mutation by Align GVGD

Classifiers according to A-GVGD, ordered from most likely to interfere with function to least likely:

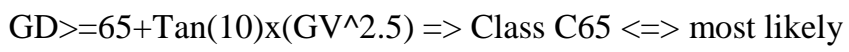

$\mathrm{GD}>=55+\operatorname{Tan}(10) \times\left(\mathrm{GV}^{\wedge} 2.0\right)=>$ Class C55

$\mathrm{GD}>=45+\operatorname{Tan}(15) \times\left(\mathrm{GV}^{\wedge} 1.7\right) \Rightarrow$ Class $\mathrm{C} 45$

$\mathrm{GD}>=35+\operatorname{Tan}(50) \times\left(\mathrm{GV}^{\wedge} 1.1\right) \Rightarrow$ Class $\mathrm{C} 35$

$\mathrm{GD}>=25+\operatorname{Tan}(55) \mathrm{x}\left(\mathrm{GV}^{\wedge} 0.95\right) \Rightarrow$ Class $\mathrm{C} 25$

$\mathrm{GD}>=15+\operatorname{Tan}(75) \times\left(\mathrm{GV}^{\wedge} 0.6\right) \Rightarrow$ Class $\mathrm{C} 15$

Else $\left(\mathrm{GD}<15+\operatorname{Tan}(75) \mathrm{x}\left(\mathrm{GV}^{\wedge} 0.6\right)\right) \Rightarrow$ Class $\mathrm{C} 0 \Leftrightarrow$ less likely

\subsection{Harmful effects of selected variants by MutPred2 server}

MutPred2 tool takes the physio-chemical properties in consideration to predict the degree of tolerance for nsSNPs. The summarized results from MutPred server for the selected 12 amino acid substitution is represented in Table 9. Gain of intrinsic disorder $(\mathrm{P}=0.0065)$ was predicted at V25G; Amino acid substitution at F51L and E56K was predicted to have a loss of proteolytic cleavage at D52 ( $\mathrm{P}=0.0095)$; at the substitution G61D, altered metal binding $(\mathrm{P}=0.0055)$ was predicted. Amino acid substitution at $\mathrm{Y} 151 \mathrm{C}$ was predicted to have loss of phosphorylation at $\mathrm{Y} 151(\mathrm{P}=0.005)$ along with altered disordered interface $(\mathrm{P}=0.0019)$. Loss of acetylation at $\mathrm{K} 161(\mathrm{P}=0.0029)$ and gain of strand $(\mathrm{P}=0.0076)$ were predicted at $\mathrm{R} 156 \mathrm{~W}$.

\begin{tabular}{lll}
\hline Amino acid variants & $\begin{array}{l}\text { Probability of deleterious amino acid } \\
\text { substitution }\end{array}$ & Top 5 features \\
\hline R19C & 0.787 & Altered disordered interface $(\mathrm{P}=0.01)$ \\
\hline G23D & 0.667 & $\begin{array}{l}\text { Altered disordered interface }(\mathrm{P}=0.03) \\
\text { Gain of intrinsic disorder }(\mathrm{P}=0.05)\end{array}$ \\
\hline V25G & 0.711 & Gain of proteolytic cleavage at $\mathrm{D} 26(\mathrm{P}=0.04)$ \\
\hline
\end{tabular}




\begin{tabular}{|c|c|c|}
\hline & & $\begin{array}{l}\text { Altered disordered interface }(\mathrm{P}=0.03) \\
\text { Gain of } \mathrm{B} \text {-factor }(\mathrm{P}=0.02)\end{array}$ \\
\hline V25L & 0.352 & N/A \\
\hline Q29P & 0.627 & N/A \\
\hline F51L & 0.642 & $\begin{array}{l}\text { Altered metal binding }(\mathrm{P}=0.03) \\
\text { Loss of proteolytic cleavage at } \mathrm{D} 52 \\
(\mathrm{P}=0.0095)\end{array}$ \\
\hline E56K & 0.527 & $\begin{array}{l}\text { Loss of loop }(\mathrm{P}=0.02) \\
\text { Altered metal binding }(\mathrm{P}=0.01) \\
\text { Loss of proteolytic cleavage at } \mathrm{D} 52 \\
(\mathrm{P}=0.0095)\end{array}$ \\
\hline T57I & 0.473 & N/A \\
\hline G61R & 0.640 & Altered metal binding $(\mathrm{P}=0.03)$ \\
\hline G61D & 0.690 & Altered metal binding $(\mathrm{P}=0.0055)$ \\
\hline Y151C & 0.625 & $\begin{array}{l}\text { Loss of phosphorylation at } \mathrm{Y} 151(\mathrm{P}=0.005) \\
\text { Altered disordered interface }(\mathrm{P}=0.0019) \\
\text { Loss of acetylation at } \mathrm{K} 154(\mathrm{P}=0.03) \\
\text { Loss of proteolytic cleavage at } \mathrm{D} 149(\mathrm{P}=0.01)\end{array}$ \\
\hline R156W & 0.619 & $\begin{array}{l}\text { Altered disordered interface }(\mathrm{P}=0.01) \\
\text { Loss of acetylation at } \mathrm{K} 161(\mathrm{P}=0.0029) \\
\text { Loss of phosphorylation at } \mathrm{Y} 151(\mathrm{P}=0.01) \\
\text { Gain of strand }(\mathrm{P}=0.0076) \\
\text { Loss of proteolytic cleavage at } \mathrm{R} 156(\mathrm{P}=0.03) \\
\text { Altered metal binding }(\mathrm{P}=0.05)\end{array}$ \\
\hline
\end{tabular}

Table 9. Prediction of the effect of nsSNPs by MutPred server

\section{Discussion}

Non synonymous single nucleotide polymorphism in genes pose a profound impact on the functionality of a protein. The determination of the phenotypic features attributed to nsSNPs by in silico tools may bring the susceptible variants interfering with the natural function. CDKN1A gene, located chromosome 6 , codes for the protein $\mathrm{p} 21^{\mathrm{Cip} 1}$, also known as cyclin dependent kinase inhibitor 1 protein. This protein inhibits CDK2 and halts cell cycle progression. It acts as a genome guardian by triggering apoptosis upon DNA damage or stress (Georgakilas et al., 2017); this function might become the opposite if mutation in CDKN1A arises (Liu and Kwiatkowski, 2015).

In this in silico study, following the final screening using the SIFT and Polyphen-2 tool, 53 missense substitutions, out of $118 \mathrm{nsSNPs}$, was predicted to be harmful. In a study to evaluate the effect of missense variants, SIFT, Polyphen2 and SNAP tool gave better result with higher accuracy than the other to identify deleterious nsSNPs (Thusberg and Vihinen, 2009). Of these $53 \mathrm{nsSNPs}$ of this analytical study, SIFT predicted $43.39 \%(\mathrm{n}=23)$ substitutions as damaging where Polyphen-2 predicted $64.15 \%$ (34) and $52.83 \%$ (28) nsSNPs as deleterious according to HumDiv and HumVar scores, respectively. Non synonymous SNPs associated with disease were highest according to MAPP (50), followed by Predict SNP (31), SNAP (29) and PhD SNP (19). Changes in the stability upon mutation was highest according to I-Mutant 3.0 tool (46) and MUpro predicted 41 nsSNPs, out of 53 as responsible to decrease the stability of the protein. I-Mutant 3.0 was found to be the most reliable predictor to identify the changes in stability upon mutation (Khan and Vihinen, 2010). Among these 53 substitutions- R19C ( $\rightarrow T$, rs769229553), G23D (A $\rightarrow$ G, rs368128681), V25G $(\mathrm{G} \rightarrow \mathrm{T}, \mathrm{rs} 765248879), \mathrm{V} 25 \mathrm{~L}(\mathrm{C} \rightarrow \mathrm{G}, \mathrm{rs} 151314631), \mathrm{Q} 29 \mathrm{P}(\mathrm{A} \rightarrow \mathrm{C} \rightarrow \mathrm{G}, \mathrm{rs} 756383281), \mathrm{F} 51 \mathrm{~L}(\mathrm{C} \rightarrow \mathrm{T}, \mathrm{rs} 748842488)$, E56K $(A \rightarrow G, r s 753291170)$, T57I $(C \rightarrow T, r s 772419969), G 61 R(C \rightarrow G, r s 758877354), G 61 D(A \rightarrow G, r s 778199042)$, $\mathrm{Y} 151 \mathrm{C}(\mathrm{A} \rightarrow \mathrm{G}, \mathrm{rs} 199801759)$ and $\mathrm{R} 156 \mathrm{~W}(\mathrm{C} \rightarrow \mathrm{G} \rightarrow \mathrm{T}, \mathrm{rs} 374154006)$ were predicted as both deleterious and responsible for decreasing stability of the protein by all above methods. Thus, further analysis on their effect on the functionality was executed. A similar study based on the dbSNP was conducted to find the deleterious SNPs of 
BARD1 gene (Alshatwi et al., 2012). Homology model generated by the Swiss-model was taken as native model, considering its overall validation score. Homology modelling has been one of the most promising method to build reliable structures (Bordoli et al., 2008). Following energy minimization, mutant model R19C and V25G presented an increase (less favorable) in the total energy count ( $\mathrm{kcal} / \mathrm{mol})$ comparing to the native model. Total scores for Y151C and R156W could not be computed due to the limitation in the amino acid length of the model structure (62 amino acid). Only three (R19C, Y151C and R156W) out of these twelve substitutions was uncovered and occurred out of the CDI (PF02234) domain predicted by Mutation 3D algorithm. Domains are the functionally active site of a protein structure and mutations on this site may have a tremendous effect on its activity (Yang et al., 2015). Biophysical analysis by Align-GVGD proposed only two substitutions (V25L and F51L) as less confidently interfering with the function of the protein. More importantly, among these 12 highly damaging mutations- R19C, G23D, F51L, Y151C and R156W occurred at the most conserved site, based on evolutionary history, on the amino acid sequence of CDKN1A, which are predicted to be the positions interfering mostly with the function of the protein. Some molecular features of some selected substitutions revealed by MutPred2 application predicted them as being actionable or confident hypotheses that they might be deleterious or associated with disease. MutPred2 general probability of deleterious mutation score for R19C, G23D, V25G, F51L, E56K, G61R, G61D, Y151C, and R156W was 0.787, $0.667,0.711,0.642,0.527,0.640,0.690,0.625$ and 0.619 respectively. The effect of missense mutation at the molecular level was also predicted by in silico analysis in Chinese patients having congenital dyserythropoietic anaemia (CDA) (Wang et al., 2018)

\section{Conclusion}

A simulation based study employed in this study to detect the deleterious nsSNPs using many computational program has revealed, 12 amino acid substitutions (R19C, G23D, V25G, V25L, Q29P, F51L, E56K, T57I, G61R, G61D, Y151C and R156W) of CDKN1A which might be responsible for the functional discrepancies comparing to the novel wild CDKN1A gene having a major role in the regulation of cell cycle progression at G1 phase. Majority of the variants identified in this study are located on domain site and conserved region of cyclin dependent kinase inhibitor 1. In situ evaluation of this gene, and its protein cyclin dependent kinase 1 in this study, which predicted some novel deleterious non-synonymous single nucleotide polymorphisms along with their functional sites and molecular features might come in help for further in vitro study.

\section{FUNDING}

This research did not receive any specific grant from funding agencies in the public, commercial, or not-for-profit sectors.

\section{CONFLICTS OF INTEREST}

This is to certify that, from the authors, there is no conflict of interests regarding the publication of this paper.

\section{REFERENCES}

Adzhubei, I.A., Schmidt, S., Peshkin, L., Ramensky, V.E., Gerasimova, A., Bork, P., Kondrashov, A.S., Sunyaev, S.R., 2010. A method and server for predicting damaging missense mutations. Nat. Methods 7, 248-249. https://doi.org/10.1038/nmeth0410-248 
Ali, S.K., Sneha, P., Priyadharshini Christy, J., Zayed, H., George Priya Doss, C., 2017. Molecular dynamics-based analyses of the structural instability and secondary structure of the fibrinogen gamma chain protein with the D356V mutation. J. Biomol. Struct. Dyn. 35, 2714-2724.

Alshatwi, A.A., Hasan, T.N., Syed, N.A., Shafi, G., Grace, B.L., 2012. Identification of Functional SNPs in BARD1 Gene and In Silico Analysis of Damaging SNPs: Based on Data Procured from dbSNP Database. PLOS ONE 7, e43939. https://doi.org/10.1371/journal.pone.0043939

Arnold, K., Bordoli, L., Kopp, J., Schwede, T., 2006. The SWISS-MODEL workspace: a web-based environment for protein structure homology modelling. Bioinforma. Oxf. Engl. 22, 195-201. https://doi.org/10.1093/bioinformatics/bti770

Ashkenazy, H., Abadi, S., Martz, E., Chay, O., Mayrose, I., Pupko, T., Ben-Tal, N., 2016. ConSurf 2016: an improved methodology to estimate and visualize evolutionary conservation in macromolecules. Nucleic Acids Res. 44, W344-W350. https://doi.org/10.1093/nar/gkw408

Bahl, R., Arora, S., Nath, N., Mathur, M., Shukla, N.K., Ralhan, R., 2000. Novel polymorphism in p21waf1/cip1 cyclin dependent kinase inhibitor gene: association with human esophageal cancer. Oncogene 19, 323.

Bendl, J., Stourac, J., Salanda, O., Pavelka, A., Wieben, E.D., Zendulka, J., Brezovsky, J., Damborsky, J., 2014. PredictSNP: Robust and Accurate Consensus Classifier for Prediction of Disease-Related Mutations. PLoS Comput. Biol. 10. https://doi.org/10.1371/journal.pcbi.1003440

Benkert, P., Tosatto, S.C.E., Schomburg, D., 2008. QMEAN: A comprehensive scoring function for model quality assessment. Proteins 71, 261-277. https://doi.org/10.1002/prot.21715

Biasini, M., Bienert, S., Waterhouse, A., Arnold, K., Studer, G., Schmidt, T., Kiefer, F., Gallo Cassarino, T., Bertoni, M., Bordoli, L., Schwede, T., 2014. SWISS-MODEL: modelling protein tertiary and quaternary structure using evolutionary information. Nucleic Acids Res. 42, W252-258. https://doi.org/10.1093/nar/gku340

Bordoli, L., Kiefer, F., Arnold, K., Benkert, P., Battey, J., Schwede, T., 2008. Protein structure homology modeling using SWISS-MODEL workspace. Nat. Protoc. 4, 1. https://doi.org/10.1038/nprot.2008.197

Bromberg, Y., Rost, B., 2007. SNAP: predict effect of non-synonymous polymorphisms on function. Nucleic Acids Res. 35, 3823-3835. https://doi.org/10.1093/nar/gkm238

Buchan, D.W.A., Minneci, F., Nugent, T.C.O., Bryson, K., Jones, D.T., 2013. Scalable web services for the PSIPRED Protein Analysis Workbench. Nucleic Acids Res. 41, W349-W357. https://doi.org/10.1093/nar/gkt381

Capriotti, E., Calabrese, R., Casadio, R., 2006. Predicting the insurgence of human genetic diseases associated to single point protein mutations with support vector machines and evolutionary information. Bioinforma. Oxf. Engl. 22, 2729-2734. https://doi.org/10.1093/bioinformatics/btl423

Capriotti, E., Fariselli, P., Casadio, R., 2005. I-Mutant2.0: predicting stability changes upon mutation from the protein sequence or structure. Nucleic Acids Res. 33, W306-310. https://doi.org/10.1093/nar/gki375

Cheng, J., Randall, A., Baldi, P., 2006. Prediction of protein stability changes for single-site mutations using support vector machines. Proteins 62, 1125-1132. https://doi.org/10.1002/prot.20810

Dassault Systèmes, 2016. , Discovery Studio Modeling Environment. Dassault Systèmes, San Diego.

Facher, E.A., Becich, M.J., Deka, A., Law, J.C., 1997. Association between human cancer and two polymorphisms occurring together in the p21Waf1/Cip1 cyclin-dependent kinase inhibitor gene. Cancer 79, 2424-2429.

Georgakilas, A.G., Martin, O.A., Bonner, W.M., 2017. p21: A Two-Faced Genome Guardian. Trends Mol. Med. 23, 310-319. https://doi.org/10.1016/j.molmed.2017.02.001

Guex, N., Peitsch, M.C., 1997. SWISS-MODEL and the Swiss-PdbViewer: an environment for comparative protein modeling. Electrophoresis 18, 2714-2723. https://doi.org/10.1002/elps.1150181505

Hicks, S., Wheeler, D.A., Plon, S.E., Kimmel, M., 2011. Prediction of missense mutation functionality depends on both the algorithm and sequence alignment employed. Hum. Mutat. 32, 661-668.

IBM Corp., 2011. , IBM SPSS Statistics for Windows. IBM Corp., Armonk, NY.

Jones, D.T., 1999. Protein secondary structure prediction based on position-specific scoring matrices. J. Mol. Biol. 292, 195-202. https://doi.org/10.1006/jmbi.1999.3091

Källberg, M., Wang, H., Wang, S., Peng, J., Wang, Z., Lu, H., Xu, J., 2012. Template-based protein structure modeling using the RaptorX web server. Nat. Protoc. 7, 1511-1522. https://doi.org/10.1038/nprot.2012.085 
Kamaraj, B., Purohit, R., 2013. In silico screening and molecular dynamics simulation of disease-associated nsSNP in TYRP1 gene and its structural consequences in OCA3. BioMed Res. Int. 2013.

Kelley, L.A., Mezulis, S., Yates, C.M., Wass, M.N., Sternberg, M.J.E., 2015. The Phyre2 web portal for protein modeling, prediction and analysis. Nat. Protoc. 10, 845. https://doi.org/10.1038/nprot.2015.053

Khan, S., Vihinen, M., 2010. Performance of protein stability predictors. Hum. Mutat. 31, 675-684. https://doi.org/10.1002/humu.21242

Kumar, P., Henikoff, S., Ng, P.C., 2009. Predicting the effects of coding non-synonymous variants on protein function using the SIFT algorithm. Nat. Protoc. 4, 1073-1081. https://doi.org/10.1038/nprot.2009.86

Li, B., Krishnan, V.G., Mort, M.E., Xin, F., Kamati, K.K., Cooper, D.N., Mooney, S.D., Radivojac, P., 2009. Automated inference of molecular mechanisms of disease from amino acid substitutions. Bioinforma. Oxf. Engl. 25, 2744-2750. https://doi.org/10.1093/bioinformatics/btp528

Liu, Y., Kwiatkowski, D.J., 2015. Combined CDKN1A/TP53 mutation in bladder cancer is a therapeutic target. Mol. Cancer Ther. 14, 174-182. https://doi.org/10.1158/1535-7163.MCT-14-0622-T

Lovell, S.C., Davis, I.W., Arendall, W.B., de Bakker, P.I.W., Word, J.M., Prisant, M.G., Richardson, J.S., Richardson, D.C., 2003. Structure validation by $C \alpha$ geometry: $\phi, \psi$ and $C \beta$ deviation. Proteins Struct. Funct. Bioinforma. 50, 437-450. https://doi.org/10.1002/prot.10286

Mathe, E., Olivier, M., Kato, S., Ishioka, C., Hainaut, P., Tavtigian, S.V., 2006. Computational approaches for predicting the biological effect of p53 missense mutations: a comparison of three sequence analysis based methods. Nucleic Acids Res. 34, 1317-1325. https://doi.org/10.1093/nar/gkj518

Meyer, M.J., Lapcevic, R., Romero, A.E., Yoon, M., Das, J., Beltrán, J.F., Mort, M., Stenson, P.D., Cooper, D.N., Paccanaro, A., Yu, H., 2016. mutation3D: Cancer Gene Prediction Through Atomic Clustering of Coding Variants in the Structural Proteome. Hum. Mutat. 37, 447-456. https://doi.org/10.1002/humu.22963

Ng, P.C., Henikoff, S., 2006. Predicting the effects of amino acid substitutions on protein function. Annu. Rev. Genomics Hum. Genet. 7, 61-80. https://doi.org/10.1146/annurev.genom.7.080505.115630

Ng, P.C., Henikoff, S., 2003. SIFT: predicting amino acid changes that affect protein function. Nucleic Acids Res. 31, 3812-3814.

Pejaver, V., Urresti, J., Lugo-Martinez, J., Pagel, K.A., Lin, G.N., Nam, H.-J., Mort, M., Cooper, D.N., Sebat, J., Iakoucheva, L.M., Mooney, S.D., Radivojac, P., 2017. MutPred2: inferring the molecular and phenotypic impact of amino acid variants. bioRxiv 134981. https://doi.org/10.1101/134981

Powell, B.L., van Staveren, I.L., Roosken, P., Grieu, F., Berns, E.M., Iacopetta, B., 2002. Associations between common polymorphisms in TP53 and p21WAF1/Cip1 and phenotypic features of breast cancer. Carcinogenesis 23, 311-315.

Ralhan, R., Agarwal, S., Mathur, M., Wasylyk, B., Srivastava, A., 2000. Association between polymorphism in p21Waf1/Cip1 cyclin-dependent kinase inhibitor gene and human oral cancer. Clin. Cancer Res. 6, 24402447.

Ravitz, M.J., Wenner, C.E., 1997. Cyclin-dependent kinase regulation during G1 phase and cell cycle regulation by TGF-beta. Adv. Cancer Res. 71, 165-207.

Sherry, S.T., Ward, M.H., Kholodov, M., Baker, J., Phan, L., Smigielski, E.M., Sirotkin, K., 2001. dbSNP: the NCBI database of genetic variation. Nucleic Acids Res. 29, 308-311.

Stojiljkovic, M., Klaassen, K., Djordjevic, M., Sarajlija, A., Brasil, S., Kecman, B., Grkovic, S., Kostic, J., RodriguezPombo, P., Desviat, L.R., 2016. Molecular and phenotypic characteristics of seven novel mutations causing branched-chain organic acidurias. Clin. Genet. 90, 252-257.

Stone, E.A., Sidow, A., 2005. Physicochemical constraint violation by missense substitutions mediates impairment of protein function and disease severity. Genome Res. 15, 978-986. https://doi.org/10.1101/gr.3804205

Tavtigian, S.V., Deffenbaugh, A.M., Yin, L., Judkins, T., Scholl, T., Samollow, P.B., de Silva, D., Zharkikh, A., Thomas, A., 2006. Comprehensive statistical study of 452 BRCA1 missense substitutions with classification of eight recurrent substitutions as neutral. J. Med. Genet. 43, 295-305. https://doi.org/10.1136/jmg.2005.033878 
Thusberg, J., Vihinen, M., 2009. Pathogenic or not? And if so, then how? Studying the effects of missense mutations using bioinformatics methods. Hum. Mutat. 30, 703-714. https://doi.org/10.1002/humu.20938

Tracy, I., Tapper, W., Parker, A., Gardiner, A., Sadullah, S., Pratt, G., Copplestone, A., Oscier, D., Best, O.G., 2017. Type C Tp53-cdkn1a pathway dysfunction occurs independently of cdkn1a gene polymorphisms in chronic lymphocytic leukaemia and is associated with tp53 abnormalities. Br. J. Haematol. 178, 824-826.

van Gunsteren, W.F., 1996. Biomolecular Simulation: The GROMOS96 Manual and User Guide. Biomos ; Zürich.

Wang, Y., Ru, Y., Liu, G., Dong, S., Li, Y., Zhu, X., Zhang, F., Chang, Y.-Z., Nie, G., 2018. Identification of CDAN1, C15ORF41 and SEC23B mutations in Chinese patients affected by congenital dyserythropoietic anemia. Gene 640, 73-78. https://doi.org/10.1016/j.gene.2017.10.027

Wu, S., Zhang, Y., 2008. MUSTER: Improving protein sequence profile-profile alignments by using multiple sources of structure information. Proteins 72, 547-556. https://doi.org/10.1002/prot.21945

Xu, D., Zhang, Y., 2011. Improving the physical realism and structural accuracy of protein models by a two-step atomic-level energy minimization. Biophys. J. 101, 2525-2534. https://doi.org/10.1016/j.bpj.2011.10.024

Yang, F., Petsalaki, E., Rolland, T., Hill, D.E., Vidal, M., Roth, F.P., 2015. Protein Domain-Level Landscape of Cancer-Type-Specific Somatic Mutations. PLOS Comput. Biol. 11, e1004147. https://doi.org/10.1371/journal.pcbi.1004147

Yates, S.C., Zafar, A., Rabai, E.M., Foxall, J.B., Nagy, S., Morrison, K.E., Clarke, C., Esiri, M.M., Christie, S., Smith, A.D., 2015. The effects of two polymorphisms on p21cip1 function and their association with Alzheimer's disease in a population of European descent. PloS One 10, e0114050.

Zerbino, D.R., Achuthan, P., Akanni, W., Amode, M.R., Barrell, D., Bhai, J., Billis, K., Cummins, C., Gall, A., Girón, C.G., Gil, L., Gordon, L., Haggerty, L., Haskell, E., Hourlier, T., Izuogu, O.G., Janacek, S.H., Juettemann, T., To, J.K., Laird, M.R., Lavidas, I., Liu, Z., Loveland, J.E., Maurel, T., McLaren, W., Moore, B., Mudge, J., Murphy, D.N., Newman, V., Nuhn, M., Ogeh, D., Ong, C.K., Parker, A., Patricio, M., Riat, H.S., Schuilenburg, H., Sheppard, D., Sparrow, H., Taylor, K., Thormann, A., Vullo, A., Walts, B., Zadissa, A., Frankish, A., Hunt, S.E., Kostadima, M., Langridge, N., Martin, F.J., Muffato, M., Perry, E., Ruffier, M., Staines, D.M., Trevanion, S.J., Aken, B.L., Cunningham, F., Yates, A., Flicek, P., 2017. Ensembl 2018. Nucleic Acids Res. https://doi.org/10.1093/nar/gkx1098 